\title{
Modeling the variance of return intervals toward volatility prediction
}

\author{
Yan Sun*1, Guanghua Lian ${ }^{\dagger 2}$, Zudi Lu ${ }^{3}$, Jennifer Loveland ${ }^{4}$, and Isaac Blackhurst ${ }^{5}$ \\ ${ }^{1,4,5}$ Department of Mathematics \& Statistics, Utah State University, 3900 Old Main \\ Hill, Logan, Utah 84322, USA. \\ ${ }^{2}$ Morgan Stanley, 20 Bank Street, London, E14 4AD, UK \\ ${ }^{3}$ Mathematical Sciences \& S3RI-Southampton Statistical Sciences Research Institute, \\ University of Southampton, SO17 1BJ, UK
}

\begin{abstract}
Interval-valued time series has been attracting increasing interest. There have been fruitful results on mean models, but variance models largely remain unexploited. In this paper, we propose a conditional heteroskedasticity model for the return interval process, which aims at capturing the underlying variance structure. Under the general framework of random sets, the model properties are investigated. Parameters are estimated by the maximum likelihood method, and the asymptotic properties are established. Empirical application to stocks and financial indices data sets suggests that our model overall outperforms the traditional GARCH for both in-sample estimation and out-of-sample prediction of the volatility.
\end{abstract}

Keywords: interval-valued time series; stationarity; maximum likelihood estimate; random sets; price range; volatility

JEL Classification: G1

MSC code: 52A22, 37M10, 91B84

\section{Introduction}

It has been a while since interval-valued data analysis was investigated and developed. There are various models and methods built upon set arithmetics, as well as under the framework of symbolic data analysis (SDA). These works cover various topics including descriptive statistics (Billard and Diday, 2003; Billard, 2007), linear regression (Diamond, 1990; Körner and Näther, 1998; Gil et al., 2002, 2007; González-Rodríguez et al., 2007; Blanco-Fernández et al., 2011; Billard, 2007; Lima Neto and Carvalho, 2008, 2010; Wei et al., 2017), hypothesis testing (Montenegro et al., 2008; Sun, 2017), and multivariate analysis (Gioia and Lauro, 2006; D'Esposito et al., 2012; Lima Neto and Anjos, 2015). Recently, there is a growing body of literature devoted

\footnotetext{
${ }^{*}$ Corresponding author. Email: Yan.Sun@usu.edu.

${ }^{\dagger}$ Dr. Guanghua Lian did most of his work on this paper while he was a Senior Lecturer in the School of Commerce at University of South Australia, Australia.
} 
to interval-valued time series (e.g. Maia et al., 2008; Han et al., 2008, 2012). In particular, asset return interval as a special interval-valued time series is believed to contain important price fluctuation information, and thus has been increasingly investigated for volatility prediction (e.g. Hu and He, 2007; He and Hu, 2009; Arroyo et al., 2011; Yang et al., 2014; Fischer et al., 2016). Intuitively, a return interval is the range of all single point returns during a certain time period. Two formal definitions of the daily return interval are provided in Fischer et al. (2016). The first one defines the current day's return interval to include all the difference values between any price of the current day and the closing price of the previous day, and is thus called the "return-interval-from-closing-price (RICP)". This definition does not consider the price fluctuation in the previous day when calculating the current day's return. To further account for that, an alternative definition was proposed such that the return interval includes all the differences between any two prices in two consecutive days, which is consequently called the "return-interval-from-price-interval (RIPI)". Let $y_{t}(s)$ be the $(\log )$ price of an asset at time $s$ on day $t$. Meanwhile, let $y_{t}^{c}$ be the closing $(\log )$ price on day $t$. The mathematical equations of the two definitions are given as

$$
\begin{aligned}
& R_{t}^{R I C P}=\left[\min _{s}\left\{y_{t}(s)\right\}-y_{t-1}^{c}, \max _{s}\left\{y_{t}(s)\right\}-y_{t-1}^{c}\right], \\
& R_{t}^{R I P I}=\left[\min _{s}\left\{y_{t}(s)\right\}-\max _{w}\left\{y_{t-1}(w)\right\}, \max _{s}\left\{y_{t}(s)\right\}-\min _{w}\left\{y_{t-1}(w)\right\}\right] .
\end{aligned}
$$

Both intervals contain richer information about the daily volatility than the single closing-toclosing return, and their potential usefulness in volatility prediction is promising. Fischer et al. (2016) applied regression techniques for interval-valued data to build predictive models for $R_{t}^{R I C P}$, and constructed volatility forecasts based on the predicted return interval. They chose to work with $R_{t}^{R I C P}$ for two reasons: 1) The size of $R_{t}^{R I P I}$ is too large - on average twice as large as that of $R_{t}^{R I C P}$. 2) The width of $R_{t}^{R I P I}$ is highly persistent. While the size of $R_{t}^{R I P I}$ being an issue is questionable, the persistence is indeed problematic since their method has a regression nature. In general, however, the persistence of the return interval as a volatility proxy may be a good thing, as opposed to be a disadvantage, given that the volatility itself has the cluster effect. Motivated by the preceding discussion, we propose to utilize the persistence in $R_{t}^{R I P I}$ to build a variance model that directly predicts the volatility. Current results regarding interval-valued time series mainly focus on the mean models, i.e., models that aim at making prediction of the interval-valued mean. The variance model for conditional heteroskedasticity, as far as the authors are aware, largely remains an unexploited area, and this study is among the very first investigations.

The issue of assets' volatility plays an essential role in modern finance. It provides a measured variability for the asset price over a certain period of time, and is a key parameter in many financial applications such as financial derivatives pricing, risk assessment, and portfolio management. The squared return of log prices used to be the "ideal" proxy of volatility, and many return-based volatility models were proposed and, for a long time, have been very popular and successful. The celebrated ARCH (Engle, 1982) and GARCH (Bollerslev, 1986) models are examples of this type. Recently, as the high-frequency data become widely available, the traditional low-frequency (e.g., daily) return is no longer quite representative of the volatility. For 
example, a small low-frequency return does not necessarily imply low volatility, as the price may fluctuate a lot and close at a similar level to the opening. On the other hand, a big return could only be the result of a very different opening price from the previous day's closing. In fact, since closing price is only a "snapshot" among numerous prices during a day, there is nothing special about it and return need not be calculated solely based on it. Therefore, traditional return-based models, using lesser information, are likely to produce inefficient or even incorrect estimates of the volatility. With the wide availability of high-frequency data, more information can obviously be utilized. Direct use of the high-frequency data can be computationally expensive and much affected by the microstructure noises. Instead, summary statistics of the high-frequency data such as the return interval can be an efficient and effective volatility proxy. It is expected that the return interval shares some similarities with the traditional point return, thus we propose to adapt the GARCH mechanism to model the variance dynamics of the return interval $R_{t}^{R I P I}$. The resulting model, in this perspective, can be viewed as an extension of the GARCH model to allow for interval-valued return input, and hence we call it the Int-GARCH model.

Technically, our Int-GARCH model for the return interval is built based on interval statistics under the random sets framework. Our theoretical results are two-fold. We first establish that under certain conditions our Int-GARCH model achieves weak stationarity that is characterized by a time invariant mean and covariance. Then, under the assumption of weak stationarity, we define and obtain the explicit formula of the autocorrelation function (ACF) of the IntGARCH process. We propose to estimate the model parameters by the method of maximum likelihood and provide the associated asymptotic properties. Simulation shows that the results are consistent with our theoretical findings. For empirical study, we analyze several stocks and indices data that are representative of the market. Our Int-GARCH model is compared to GARCH using the RV as the market proxy. Based on both in-sample and out-of-sample comparisons, our Int-GARCH model overall outperforms GARCH with higher correlations and reduced errors to RV.

The rest of the paper is organized as follows. Random sets preliminaries are provided in Section 2. In Section 3, we formally introduce our Int-GARCH model and its usage for volatility prediction. Stationarity is derived and presented in Section 4. Section 5 discusses the maximum likelihood estimator for the model parameters and carefully investigates its performances by a simulation study. Empirical study with the stocks and indices data, as well as a detailed discussion, are reported in Section 6. We finish with concluding remarks in Section 7. Proofs of the theorems are deferred to the Appendix. Proofs of corollaries, along with all the useful lemmas, are provided in the online Supplementary Appendix A.

\section{Preliminaries of random sets}

For ease of notation, we will denote $r_{t}=R_{t}^{R I P I}$. Throughout the paper, $r_{t}$ is viewed as a random interval and its dynamics is modeled under the framework of random sets. To facilitate our presentation, we briefly introduce the basic notations and definitions in the random set theory. For more details we refer the readers to Kendall (1974), Matheron (1975), Artstein and Vitale (1975), Molchanov (2005), Sun and Ralescu (2015), among others. 
Let $(\Omega, \mathcal{L}, P)$ be a probability space. Denote by $\mathcal{K}\left(\mathbb{R}^{d}\right)$ or $\mathcal{K}$ the collection of all non-empty compact subsets of $\mathbb{R}^{d}$. A random compact set is a Borel measurable function $A: \Omega \rightarrow \mathcal{K}$, $\mathcal{K}$ being equipped with the Borel $\sigma$-algebra induced by the Hausdorff metric $\rho_{H}$. As a metric space, $\left(\mathcal{K}, \rho_{H}\right)$ is complete and separable (Debreu, 1967). In the space $\mathcal{K}$, a linear structure is defined by Minkowski addition and scalar multiplication, i.e.,

$$
A+B=\{a+b: a \in A, b \in B\}, \quad \lambda A=\{\lambda a: a \in A\},
$$

$\forall A, B \in \mathcal{K}$ and $\lambda \in \mathbb{R}$. However, $\mathcal{K}$ is not a linear space, due to the lack of an inverse element of addition.

If $A(\omega)$ is convex almost surely, then $A$ is called a random compact convex set. Especially, a one-dimensional random compact convex set is called a random interval. Let $\mathcal{K}_{\mathcal{C}}\left(\mathbb{R}^{d}\right)$ denote the space of non-empty compact convex subsets of $\mathbb{R}^{d}$. According to the embedding theorems (Ràdström, 1952; Hörmander, 1954), $\mathcal{K}_{\mathcal{C}}$ can be embedded isometrically into the Banach space $C(S)$ of continuous functions on the unit sphere $S^{d-1}$. These functions are realized by the support function of $A \in \mathcal{K}_{\mathcal{C}}$ defined on $S^{d-1}$ as:

$$
s_{A}(u)=\sup _{a \in A}\langle u, a\rangle, \quad \forall u \in S^{d-1} .
$$

As a result, a compact convex set can be represented by its support function, and an $L_{2}$ metric in $\mathcal{K}_{\mathcal{C}}\left(\mathbb{R}^{d}\right)$ is given via the support function by

$$
\rho_{2}(A, B)=\left\|s_{A}-s_{B}\right\|_{2}=\left[d \int_{S^{d-1}}\left|s_{A}(u)-s_{B}(u)\right|^{2} \mu(d u)\right]^{\frac{1}{2}}, \quad \forall A, B \in \mathcal{K}_{\mathcal{C}} .
$$

It is known that $\rho_{H}$ and $\rho_{2}$ are equivalent metrics, but $\rho_{2}$ is more preferred for statistical inferences, due to many of its established properties (Körner, 1995, 1997). When $d=1$, the unit sphere $S^{0}=\{1,-1\}$, and the support function for an interval $x \in \mathcal{K}_{\mathcal{C}}(\mathbb{R})$ is

$$
s_{x}(u)= \begin{cases}x^{U}, & u=1 \\ -x^{L}, & u=-1\end{cases}
$$

where $(\cdot)^{U}$ and $(\cdot)^{L}$ denote the upper and lower bounds, respectively. Then, the $\rho_{2}$-distance between two intervals $x$ and $y$ is calculated to be

$$
\begin{aligned}
\rho_{2}(x, y) & =\left[\frac{1}{2}\left(s_{x}(-1)-s_{x}(-1)\right)^{2}+\frac{1}{2}\left(s_{x}(1)-s_{y}(1)\right)^{2}\right]^{\frac{1}{2}} \\
& =\left[\frac{1}{2}\left(x^{L}-y^{L}\right)^{2}+\frac{1}{2}\left(x^{U}-y^{U}\right)^{2}\right]^{\frac{1}{2}} \\
& =\left[\left(x^{C}-y^{C}\right)^{2}+\left(x^{R}-y^{R}\right)^{2}\right]^{\frac{1}{2}}
\end{aligned}
$$

where $(\cdot)^{C}$ and $(\cdot)^{R}$ denote the corresponding center and radius, respectively.

For a random set $X \in \mathcal{K}_{\mathcal{C}}$, the well accepted expectation is defined by the Aumann integral 
of set-valued function (Aumann, 1965) as

$$
\mathrm{E}(X)=\{\mathrm{E} \xi: \xi \in X \text { a.s., } \mathrm{E}\|\xi\|<\infty\} .
$$

Alternatively, Fréchet (Fréchet, 1948) gave a general definition for the expectation of a random element $X$ in the metric space $\left(\mathcal{K}_{\mathcal{C}}\left(\mathbb{R}^{d}\right), \rho\right)$, known as the Fréchet expectation, as the solution of

$$
\mathrm{E} \rho^{2}\left(X, \mathrm{E}_{F}(X)\right)=\inf _{A \in \mathcal{K}_{\mathcal{C}}} \mathrm{E}^{2}(X, A)
$$

Notice that equation (4) is fulfilled by the random variable w.r.t. Euclidean metric in $\mathbb{R}$. The spirit of Fréchet expectation lies in that it extends this principle to $\left(\mathcal{K}_{\mathcal{C}}\left(\mathbb{R}^{d}\right), \rho\right)$. In addition, it naturally yields the variance of $X$ as $\mathrm{E}^{2}\left(X, \mathrm{E}_{F}(X)\right)$. If we choose $\rho$ to be $\rho_{2}$, the Fréchet expectation $\mathrm{E}_{F}(X)$ coincides with the Aumann expectation $\mathrm{E}(X)$, and the variance is

$$
\operatorname{Var}(X)=\mathrm{E} \rho_{2}^{2}(X, \mathrm{E}(X))
$$

See Lyashenko (1982), Körner (1995), and Körner (1997). Restricting to $d=1$, the Aumann expectation of a random interval $X$ is simply

$$
\mathrm{E}(X)=\left[\mathrm{E}\left(X^{C}\right)-\mathrm{E}\left(X^{R}\right), \mathrm{E}\left(X^{C}\right)+\mathrm{E}\left(X^{R}\right)\right]
$$

and the variance of $X$ is consequently calculated as

$$
\operatorname{Var}(X)=\mathrm{E}\left[\left(X^{C}-\mathrm{E}\left(X^{C}\right)\right)^{2}+\left(X^{R}-\mathrm{E}\left(X^{R}\right)\right)^{2}\right]=\operatorname{Var}\left(X^{C}\right)+\operatorname{Var}\left(X^{R}\right) .
$$

Let $\mathcal{F}$ be any $\sigma$-filed on $\Omega$. The conditional expectation of a random set given $\mathcal{F}$ is defined by the integral and conditional expectation of multivalued functions (Hiai and Umegaki, 1977). Specifically for a random interval $X$,

$$
\mathrm{E}(X \mid \mathcal{F})=\left[\mathrm{E}\left(X^{C} \mid \mathcal{F}\right)-\mathrm{E}\left(X^{R} \mid \mathcal{F}\right), \mathrm{E}\left(X^{C} \mid \mathcal{F}\right)+\mathrm{E}\left(X^{R} \mid \mathcal{F}\right)\right]
$$

Based on the conditional expectation, the conditional variance of a random set is defined as the conditional mean squared distance from its conditional expectation (Näther and Wünsche, 2007). According to the definition, the conditional variance of $X$ with respect to the distance $\rho_{2}$ is given by

$$
\begin{aligned}
\operatorname{Var}(X \mid \mathcal{F}) & =E\left[\rho_{2}^{2}(X, E(X \mid \mathcal{F})) \mid \mathcal{F}\right] \\
& =E\left[\left(X^{C}-E(X \mid \mathcal{F})^{C}\right)^{2}+\left(X^{R}-E(X \mid \mathcal{F})^{R}\right)^{2} \mid \mathcal{F}\right] \\
& =E\left[\left(X^{C}-E\left(X^{C} \mid \mathcal{F}\right)\right)^{2}+\left(X^{R}-E\left(X^{R} \mid \mathcal{F}\right)\right)^{2} \mid \mathcal{F}\right] \\
& =E\left[\left(X^{C}-E\left(X^{C} \mid \mathcal{F}\right)\right)^{2} \mid \mathcal{F}\right]+E\left[\left(X^{R}-E\left(X^{R} \mid \mathcal{F}\right)\right)^{2} \mid \mathcal{F}\right] \\
& =\operatorname{Var}\left(X^{C} \mid \mathcal{F}\right)+\operatorname{Var}\left(X^{R} \mid \mathcal{F}\right) .
\end{aligned}
$$




\section{The Int-GARCH model}

\subsection{Model specification}

We assume observing a return interval process $\left\{r_{t}\right\}_{t=1}^{T}$ of the form

$$
r_{t}=\left[\lambda_{t}-\delta_{t}, \lambda_{t}+\delta_{t}\right], t=1,2, \cdots, T \text {. }
$$

That is, $\left\{\lambda_{t}\right\}_{t=1}^{T}$ and $\left\{\delta_{t}\right\}_{t=1}^{T}$ are the associated center and radius processes, both of which are observable. According to (5) and (6), the mean and variance of $r_{t}$ as a random interval are

$$
\begin{aligned}
\mathrm{E}\left(r_{t}\right) & =\left[\mathrm{E}\left(\lambda_{t}\right)-\mathrm{E}\left(\delta_{t}\right), \mathrm{E}\left(\lambda_{t}\right)+\mathrm{E}\left(\delta_{t}\right)\right] \\
\operatorname{Var}\left(r_{t}\right) & =\operatorname{Var}\left(\lambda_{t}\right)+\operatorname{Var}\left(\delta_{t}\right) .
\end{aligned}
$$

Let $\mathcal{F}_{t}$ denote the information set up to time $t$, i.e. $\mathcal{F}_{t}=\sigma\left\{r_{s}: s \leq t\right\}$. We are concerned with the conditional variance $H_{t}^{2}$ of $r_{t}$ given $\mathcal{F}_{t-1}$. According to (8), $H_{t}^{2}$ with respect to $\rho_{2}$ is computed as

$$
H_{t}^{2}=\operatorname{Var}\left(r_{t} \mid \mathcal{F}_{t-1}\right)=\operatorname{Var}\left(\lambda_{t} \mid \mathcal{F}_{t-1}\right)+\operatorname{Var}\left(\delta_{t} \mid \mathcal{F}_{t-1}\right) .
$$

The GARCH model depicts the conditional variance of a point-valued return process as a linear function of the past squared returns and variances. This was inspired by the fact that assets returns usually exhibit volatility clustering: large variations in prices tend to cluster together, resulting in separate dynamic and tranquil periods of the market. Extending this spirit to the interval-valued process $\left\{r_{t}\right\}$, one would expect, conceptually, a model like

$$
H_{t}^{2}=g\left(H_{s}^{2}, \hat{H}_{s}^{2}\left(r_{s}\right): s \leq t-1\right)
$$

where $\hat{H}_{s}^{2}\left(r_{s}\right)$ denotes a return range based proxy for $H_{s}^{2}$, and $g$ is linear in $H_{s}^{2}$ and $\hat{H}_{s}^{2}\left(r_{s}\right)$. Assuming $\mathrm{E}\left(\lambda_{t} \mid \mathcal{F}_{t-1}\right)=0$ and $\mathrm{E}\left(\delta_{t} \mid \mathcal{F}_{t-1}\right)=c>0$, a natural form of $\hat{H}_{s}^{2}\left(r_{s}\right)$ is

$$
\hat{H}_{s}^{2}\left(r_{s}\right)=\lambda_{s}^{2}+\delta_{s}^{2}-c^{2}
$$

where $\lambda_{s}^{2}$ and $\delta_{s}^{2}-c^{2}$ are proxies for $\operatorname{Var}\left(\lambda_{s} \mid \mathcal{F}_{s-1}\right)$ and $\operatorname{Var}\left(\delta_{s} \mid \mathcal{F}_{s-1}\right)$, respectively. Thus, a reasonable function $g$ in (11) seems to imply

$$
H_{t}^{2}=\mu+\sum_{i=1}^{p} \alpha_{i}\left[\lambda_{t-i}^{2}+\delta_{t-i}^{2}-c^{2}\right]+\sum_{i=1}^{q} \beta_{i} H_{t-i}^{2}
$$

where $p>0, q \geq 0$. The constant $c$ can be absorbed into the parameter $\mu$ and the above equation is simplified to

$$
H_{t}^{2}=\mu+\sum_{i=1}^{p} \alpha_{i}\left[\lambda_{t-i}^{2}+\delta_{t-i}^{2}\right]+\sum_{i=1}^{q} \beta_{i} H_{t-i}^{2} .
$$

To give more flexibility to our model, we allow for different degrees of dependence of $H_{t}$ on the past centers and radii. In addition, adopting the idea of more robust modeling of volatility by Taylor (1986) and Schwert (1990), we propose to model the conditional standard deviation $H_{t}$ 
directly, instead of via the conditional variance $H_{t}^{2}$ (Ding et al. (1993) also considered such a specification as a special case of their A-PARCH model).

Given the above discussion, our Int-GARCH $(p, q, w)$ model for the return interval process $r_{t}=\left[\lambda_{t}-\delta_{t}, \lambda_{t}+\delta_{t}\right]$ is specified as

$$
\begin{aligned}
& r_{t}=h_{t} \cdot v_{t}, \\
& v_{t}=\left[\epsilon_{t}-\eta_{t}, \epsilon_{t}+\eta_{t}\right], \\
& \epsilon_{t} \stackrel{i . i . d .}{\sim} N(0,1), \\
& \eta_{t} \stackrel{i . i . d .}{\sim} \Gamma(k, 1), \\
& h_{t}=\mu+\sum_{i=1}^{p} \alpha_{i}\left|\lambda_{t-i}\right|+\sum_{i=1}^{q} \beta_{i} \delta_{t-i}+\sum_{i=1}^{w} \gamma_{i} h_{t-i},
\end{aligned}
$$

where $\lambda_{t}$ and $\delta_{t}$ are the center and the radius of $r_{t}$, respectively, $p>0, q>0, w \geq 0$, and $\left\{\alpha_{i}\right\}_{i=1}^{p}$, $\left\{\beta_{i}\right\}_{i=1}^{q},\left\{\gamma_{i}\right\}_{i=1}^{w}$ are all positive constants. In addition, the error terms $\epsilon_{t}$ and $\eta_{t}$ are assumed to be independent. In (12), "." denotes the scalar multiplication. Under this specification, the conditional variance of $r_{t}$ is seen to be

$$
H_{t}^{2}=\operatorname{Var}\left(h_{t} \epsilon_{t} \mid \mathcal{F}_{t-1}\right)+\operatorname{Var}\left(h_{t} \eta_{t} \mid \mathcal{F}_{t-1}\right)=h_{t}^{2}(1+k)
$$

Although we impose parametric assumptions on the random errors $\epsilon_{t}$ and $\eta_{t}$ to simplify our presentation here, they are not really necessary. In practice, it is best to use the true data generating distributions, which vary from data to data. So, in replacement of (14)-(15), a relaxed yet sufficient specification for $\epsilon_{t}$ and $\eta_{t}>0$ is

$$
\begin{aligned}
& \operatorname{Var}\left(\epsilon_{t} \mid \mathcal{F}_{t-1}^{\epsilon, \eta}\right)=1, \\
& \operatorname{Var}\left(\eta_{t} \mid \mathcal{F}_{t-1}^{\epsilon, \eta}\right)=k .
\end{aligned}
$$

Remark 1. Alternatively, equation (16) can be specified by the variance as

$$
h_{t}^{2}=\mu+\sum_{i=1}^{p} \alpha_{i} \lambda_{t-i}^{2}+\sum_{i=1}^{q} \beta_{i} \delta_{t-i}^{2}+\sum_{i=1}^{w} \gamma_{i} h_{t-i}^{2} .
$$

Additional to the robustness concern that we mentioned previously, another important reason we chose (16) is that it allows for convenient calculation of odd moments. A drawback of the variance specification (18) is the complication to calculate odd moments due to the need of a fraction power of $h_{t}^{2}$. For the GARCH model, this is probably fine, as the odd moments are all equal to 0 by symmetry. However, odd moments of the Int-GARCH model are intervals centered at 0 , whose length or radius would be difficult to compute with the variance specification.

Remark 2. A more general metric for $\mathcal{K}_{\mathcal{C}}(\mathbb{R})$ was proposed by Gil et al. (2001), which essentially 
takes the form

$$
\rho_{W}^{2}(x, y)=\left(x^{C}-y^{C}\right)^{2}+\left(x^{R}-y^{R}\right)^{2} \int_{[0,1]}(2 \lambda-1)^{2} d W(\lambda), \quad x, y \in \mathcal{K}_{\mathcal{C}}(\mathbb{R})
$$

where $W$ is any non-degenerate symmetric measure on $[0,1]$. Compared to the $\rho_{2}$ metric in (2), the flexibility of $\rho_{W}$ lies in its choice of a weight between the center and radius. Since we use different parameters $\alpha$ 's and $\beta$ 's for the center and radius, respectively, this flexibility is in fact accounted for in our specification of $h_{t}$ in (16).

\subsection{Volatility forecasting}

The $h_{t}$ in the model (12)-(16) relates to the conditional standard deviation $H_{t}$ of the return range $r_{t}$ according to equation (17). However, $H_{t}$ is not exactly the daily volatility as in the literature. To see the relation between $h_{t}$ and the daily volatility $\sigma_{t}^{2}$, we notice that $r_{t}$ contains all possible returns of day $t$, using different prices during days $t$ and $t-1$. Consider an arbitrary return at position $\omega$ in $r_{t}$, denoted by $r_{t}(\omega)$, as the volatility proxy, i.e.

$$
r_{t}(\omega)=h_{t}\left(\epsilon_{t}+\omega \eta_{t}\right), \omega \in[-1,1]
$$

Volatility based on $r_{t}(\omega)$ is calculated as

$$
\sigma_{t}^{2}(\omega)=\operatorname{Var}\left(r_{t}(\omega) \mid \mathcal{F}_{t-1}\right)=\left(1+\omega^{2} k\right) h_{t}^{2}
$$

Our Int-GARCH volatility $\sigma_{t}^{2}$ is defined as the average of $\left\{\sigma_{t}^{2}(\omega): \omega \in[-1,1]\right\}$. Assuming equal weight for each point return, $\sigma_{t}^{2}$ can be calculated as

$$
\sigma_{t}^{2}=\frac{\int_{-1}^{1} \sigma_{t}^{2}(\omega) d(\omega)}{\int_{-1}^{1} d(\omega)}=\frac{1}{2} \int_{-1}^{1}\left(1+\omega^{2} k\right) h_{t}^{2} d(\omega)=\left(1+\frac{1}{3} k\right) h_{t}^{2}
$$

Therefore, volatility forecast is essentially made by predicting $h_{t}$. The 1 -step-ahead prediction of $h_{t}$ is immediately defined by equation (16) as

$$
\hat{h}_{t}(1)=\mu+\sum_{i=1}^{p} \alpha_{i}\left|\lambda_{t+1-i}\right|+\sum_{i=1}^{q} \beta_{i} \delta_{t+1-i}+\sum_{i=1}^{w} \gamma_{i} h_{t+1-i} .
$$

To make a 2-step-ahead prediction, notice that

$$
\begin{aligned}
h_{t+2} & =\mu+\sum_{i=1}^{p} \alpha_{i}\left|\lambda_{t+2-i}\right|+\sum_{i=1}^{q} \beta_{i} \delta_{t+2-i}+\sum_{i=1}^{w} \gamma_{i} h_{t+2-i} \\
& =\mu+\left(\alpha_{1}\left|\epsilon_{t+1}\right|+\beta_{1} \eta_{t+1}+\gamma_{1}\right) h_{t+1}+\sum_{i=2}^{p} \alpha_{i}\left|\lambda_{t+2-i}\right|+\sum_{i=2}^{q} \beta_{i} \delta_{t+2-i}+\sum_{i=2}^{w} \gamma_{i} h_{t+2-i}
\end{aligned}
$$


Replacing $\left|\epsilon_{t+1}\right|$ and $\eta_{t+1}$ with their expectations, and $h_{t+1}$ with $\hat{h}_{t}(1)$, we get

$$
\hat{h}_{t}(2)=\mu+\left(\alpha_{1} \sqrt{\frac{2}{\pi}}+\beta_{1} k+\gamma_{1}\right) \hat{h}_{t}(1)+\sum_{i=2}^{p} \alpha_{i}\left|\lambda_{t+2-i}\right|+\sum_{i=2}^{q} \beta_{i} \delta_{t+2-i}+\sum_{i=2}^{w} \gamma_{i} h_{t+2-i} .
$$

The general $l$-step-ahead prediction can be calculated recursively. In particular, the formula for Int-GARCH $(1,1,1)$ model is given by

$$
\hat{h}_{t}(l)=\mu+\left(\alpha_{1} \sqrt{\frac{2}{\pi}}+\beta_{1} k+\gamma_{1}\right) \hat{h}_{t}(l-1), l>1 .
$$

\section{Stationarity and autocorrelation}

\subsection{Stationarity}

We provide the necessary and sufficient conditions of mean stationarity for the Int-GARCH $(p, q, w)$ model in the following theorem.

Theorem 1. Consider the general Int-GARCH model (12)-(16). Define

$$
x_{i, t}=\alpha_{i}\left|\epsilon_{t}\right| I_{\{1 \leq i \leq p\}}+\beta_{i} \eta_{t} I_{\{1 \leq i \leq q\}}+\gamma_{i} I_{\{1 \leq i \leq w\}}
$$

and

$$
E\left(x_{i, t}\right)=\mu_{i}
$$

where $i=1,2, \cdots, m=\max \{p, q, w\}$. Assume $\left\{r_{t}\right\}$ starts from its infinite past with a finite mean. Then, $E\left(h_{t}\right)<\infty$ if and only if $\sum_{i=1}^{m} \mu_{i}<1$. When this condition is satisfied,

$$
E h_{t}=\frac{\mu}{1-\sum_{i=1}^{m} \mu_{i}}
$$

and

$$
E r_{t}=\left[-k E\left(h_{t}\right), k E\left(h_{t}\right)\right] .
$$

It is derived in Körner (1995) that the covariance between two random intervals with respect to the $\rho_{2}$ metric is the sum of the covariances between the two centers and two radii. This implies

$$
\operatorname{Cov}\left(r_{t}, r_{s}\right)=\operatorname{Cov}\left(\lambda_{t}, \lambda_{s}\right)+\operatorname{Cov}\left(\delta_{t}, \delta_{s}\right), s, t \in \mathbb{N}
$$

We are ready to extend the notion of weak stationarity to interval-valued time series in the obvious way.

Definition 1. An interval-valued time series $\left\{r_{t}\right\}$ is said to be weakly stationary, or secondmoment stationary, if its unconditional mean $E\left(r_{t}\right)$ and covariance $\operatorname{Cov}\left(r_{t}, r_{t+s}\right)$ exist and are independent of time $t$ for all integers $s$, where $E\left(r_{t}\right)$ and $\operatorname{Cov}\left(r_{t}, r_{t+s}\right)$ are given in (9) and (30), respectively.

The existences of $\mathrm{E}\left(r_{t}\right)$ and $\operatorname{Var}\left(r_{t}\right)$ are equivalent to the finiteness of $\mathrm{E}\left(h_{t}\right)$ and $\mathrm{E}\left(h_{t}^{2}\right)$, respectively. That being said, $\mathrm{E}\left(h_{t}^{2}\right)<\infty$ implies the existence of the first two moments of $r_{t}$, 
which in light of the inequality

$$
\left|\operatorname{Cov}\left(r_{t}, r_{t+h}\right)\right| \leq\left|\operatorname{Cov}\left(\lambda_{t}, \lambda_{t+h}\right)\right|+\left|\operatorname{Cov}\left(\delta_{t}, \delta_{t+h}\right)\right| \leq \operatorname{Var}\left(\lambda_{t}\right)+\operatorname{Var}\left(\delta_{t}\right),
$$

means weak stationarity essentially. We thus give precise conditions for the weak stationarity of second-order moments for Int-GARCH$(p, q, w)$ as follows.

Theorem 2. Consider the Int-GARCH $(p, q, w)$ model $\left\{r_{t}\right\}$. Assume $\left\{r_{t}\right\}$ starts from its infinite past with a finite variance. Define

$$
A_{t}=\left[\begin{array}{ccccc}
x_{1, t-1} & x_{2, t-2} & \cdots & x_{m-1, t-m+1} & x_{m, t-m} \\
1 & 0 & \cdots & 0 & 0 \\
\cdots & \cdots & \cdots & \cdots & \cdots \\
0 & 0 & \cdots & 1 & 0
\end{array}\right]
$$

with $x_{i, t}, i=1, \cdots, m$, defined in (26). Then, $E\left(h_{t}^{2}\right)<\infty$ if and only if the eigenvalues of the matrix $E\left(A_{t} \otimes A_{t}\right)$ are within a unit circle, where $\otimes$ stands for the Kronecker product.

Remark 3. As we mentioned previously, alternative equations can be used to specify the conditional heteroskedesticity. If the variance specification (18) is employed and we define

$$
\tilde{x}_{i, t}=\alpha_{i} \epsilon_{t}^{2} I_{\{1 \leq i \leq p\}}+\beta_{i} \eta_{t}^{2} I_{\{1 \leq i \leq q\}}+\gamma_{i} I_{\{1 \leq i \leq w\}},
$$

then a similar argument as Theorem 1 yields that $E\left(h_{t}\right)^{2}<\infty$ if and only if $\sum_{i=1}^{m} \tilde{\mu}_{i}<1$, where $\tilde{\mu}_{i}=E\left(\tilde{x}_{i, t}\right)$. In view of Definition 1, this essentially provides an equivalent condition for weak stationarity, which is a convenience of the variance specification.

Similar to the traditional GARCH model, the Int-GARCH $(1,1,1)$ process is a simple but effective model for analyzing interval-valued time series with conditional heteroskedasticity. In this section, we derive several important distributional properties of Int-GARCH(1,1,1). Before we present our theoretical results, notice that for the $\operatorname{Int}-\mathrm{GARCH}(1,1,1)$ process,

$$
\begin{aligned}
h_{t} & =\mu+\alpha_{1}\left|\lambda_{t-1}\right|+\beta_{1} \delta_{t-1}+\gamma_{1} h_{t-1} \\
& =\mu+\alpha_{1}\left|\epsilon_{t-1}\right| h_{t-1}+\beta_{1} \eta_{t-1} h_{t-1}+\gamma_{1} h_{t-1} \\
& =\mu+\left(\alpha_{1}\left|\epsilon_{t-1}\right|+\beta_{1} \eta_{t-1}+\gamma_{1}\right) h_{t-1} .
\end{aligned}
$$

Defining the i.i.d. random variables $x_{t}=\alpha_{1}\left|\epsilon_{t-1}\right|+\beta_{1} \eta_{t-1}+\gamma_{1}, t \in \mathbb{N}, h_{t}$ can be written by the recursive equation

$$
h_{t}=\mu+x_{t} h_{t-1} .
$$

We will use (33) throughout this section. Immediately following Theorem 1, mean stationarity of the Int-GARCH$(1,1,1)$ can be established as the special case of $p=q=w=1$. We state this result in the following Corollary 1.

Corollary 1. Consider the Int-GARCH model (12)-(16) with $p=q=w=1$. Assume $\left\{r_{t}\right\}$ 
starts from its infinite past with a finite mean. Then, $E\left(h_{t}\right)<\infty$ if and only if $E\left(x_{t}\right)<1$, i.e.

$$
\alpha_{1} \sqrt{\frac{2}{\pi}}+\beta_{1} k+\gamma_{1}<1
$$

When this condition is satisfied,

$$
E\left(h_{t}\right)=\frac{\mu}{1-\alpha_{1} \sqrt{2 / \pi}-\beta_{1} k-\gamma_{1}},
$$

and

$$
E\left(r_{t}\right)=\left[-k E\left(h_{t}\right), k E\left(h_{t}\right)\right] .
$$

Second-moment stationarity for Int-GARCH $(1,1,1)$ can also be stated more explicitly and is given in the following.

Corollary 2. Consider the Int-GARCH(1,1,1) model $\left\{r_{t}\right\}$. Assume $\left\{r_{t}\right\}$ starts from its infinite past with a finite variance. Then $E\left(h_{t}^{2}\right)<\infty$ if and only if $E\left(x_{t}^{2}\right)<1$ (with $x_{t}$ defined in (33)), i.e.

$$
\alpha_{1}^{2}+\beta_{1}^{2}\left(k+k^{2}\right)+\gamma_{1}^{2}+2 \alpha_{1} \beta_{1} \sqrt{\frac{2}{\pi}} k+2 \alpha_{1} \gamma_{1} \sqrt{\frac{2}{\pi}}+2 \beta_{1} \gamma_{1} k<1
$$

When this condition is satisfied,

$$
E\left(h_{t}^{2}\right)=\mu^{2} \frac{C_{1}+1}{\left(C_{2}-1\right)\left(C_{1}-1\right)},
$$

and

$$
\operatorname{Var}\left(r_{t}\right)=\left(1+k+k^{2}\right) E\left(h_{t}^{2}\right)-k^{2}\left[E\left(h_{t}\right)\right]^{2},
$$

where $E\left(h_{t}\right)$ is given in (34), and $C_{1}=E\left(x_{t}\right), C_{2}=E\left(x_{t}^{2}\right)$.

Corollary 3. The Int-GARCH(1,1,1) process is weakly stationary, or second-moment stationary, if and only if $E\left(x_{t}^{2}\right)<1$.

\subsection{Autocorrelation}

The autocorrelation for a general Int-GARCH model is rather involved. So we focus on the covariance function for the practically most important Int-GARCH $(1,1,1)$ model here, which, under weak stationarity, is given in Theorem 3 below. From Theorem 3 the auto-correlation function $(\mathrm{ACF})$ for the Int-GARCH$(1,1,1)$ is derived in Corollary 4.

Theorem 3. Consider the Int-GARCH(1,1,1) process $\left\{r_{t}\right\}$. Under the assumptions of Corollary 2, the covariance of any two random intervals $r_{t}$ and $r_{t+s}$ is given by

$$
\operatorname{Cov}\left(r_{t}, r_{t+s}\right)= \begin{cases}\left(1+k+k^{2}\right) E\left(h_{t}^{2}\right)-k^{2}\left[E\left(h_{t}\right)\right]^{2}, & s=0 \\ k E\left(h_{t} h_{t+s} \eta_{t}\right)-k^{2}\left[E\left(h_{t}\right)\right]^{2}, & |s|>0\end{cases}
$$


where $E\left(h_{t}\right)$ and $E\left(h_{t}^{2}\right)$ are given in (34) and (36), respectively, and $E\left(h_{t} h_{t+s} \eta_{t}\right)$ is calculated explicitly in Lemma 1 (see Appendix).

The notion of the variance and covariance for compact convex random sets were naturally extended to the correlation coefficient of two random sets $A$ and $B$, which is defined as

$$
\operatorname{Corr}(A, B)=\frac{\operatorname{Cov}(A, B)}{\sqrt{\operatorname{Var}(A) \operatorname{Var}(B)}}
$$

Based on this definition, the auto-correlation function of the Int-GARCH $(1,1,1)$ process is immediately calculated.

Corollary 4. Under the assumptions of Corollary 2, the auto-correlation function of the Int$\operatorname{GARCH}(1,1,1)$ process $\left\{r_{t}\right\}$ is

$$
\rho(s)= \begin{cases}1, & s=0 \\ \frac{k E\left(h_{t} h_{t+s} \eta_{t}\right)-k^{2}\left[E\left(h_{t}\right)\right]^{2}}{\left(1+k+k^{2}\right) E\left(h_{t}^{2}\right)-k^{2}\left[E\left(h_{t}\right)\right]^{2}}, & |s|>0 .\end{cases}
$$

The ACF for a specific Int-GARCH $(1,1,1)$ model (Model I in the simulation) is plotted in Figure 1. We see that the centers are uncorrelated. This has been verified by (50) in the proof of Theorem 3. The radii, or the lengths of the intervals, have a relatively persistent auto-correlation, which coincides with the phenomenon of "volatility clustering". This long-term dependence of radii carries over to the intervals as a whole, and results in a slow-dying ACF of the interval-valued process.

\section{Figure 1 is approximately here.}

\section{Parameter estimation}

In this section, we develop parameter estimation of our Int-GARCH model and the asymptotic properties of the estimators. There are two groups of parameters: the error distribution parameter $k>0$ and the variance parameters

$$
\boldsymbol{\theta}=\left[\mu, \alpha_{1}, \cdots, \alpha_{p}, \beta_{1}, \cdots, \beta_{q}, \gamma_{1}, \cdots, \gamma_{w}\right]^{\prime}
$$

under which we denote $h_{t}=h_{t}(\boldsymbol{\theta})$ for clarity when needed, where' stands for the transpose of a vector or matrix. We provide joint estimations of $k$ and $\boldsymbol{\theta}$ by the method of maximum likelihood, and the asymptotic normality for the joint MLE is established. A simulation study is presented that shows the empirical performance of the estimators. 


\subsection{The joint MLE of $k$ and $\theta$}

Conditioning on $\mathcal{F}_{0}$, the likelihood function of the data $\left\{r_{t}, t=1, \cdots, T\right\}$ is

$$
\begin{aligned}
L(\boldsymbol{\theta} ; k) \mid \mathcal{F}_{0} & =\prod_{t=1}^{T} f\left(\lambda_{t}, \delta_{t} \mid \mathcal{F}_{t-1}\right)=\prod_{t=1}^{T} f\left(\lambda_{t} \mid \mathcal{F}_{t-1}\right) f\left(\delta_{t} \mid \mathcal{F}_{t-1}\right) \\
& =\prod_{t=1}^{T} \frac{1}{h_{t} \sqrt{2 \pi}} e^{-\frac{\lambda_{t}^{2}}{2 h_{t}^{2}}} \frac{1}{\Gamma(k) h_{t}^{k}} \delta_{t}^{k-1} e^{-\frac{\delta_{t}}{h_{t}}} \\
& =\prod_{t=1}^{T} \frac{\delta_{t}^{k-1}}{\sqrt{2 \pi} \Gamma(k)} h_{t}^{-(k+1)} e^{-\frac{\lambda_{t}^{2}}{2 h_{t}^{2}}-\frac{\delta_{t}}{h_{t}}} \\
& \propto \prod_{t=1}^{T} \delta_{t}^{k-1} \Gamma^{-1}(k) h_{t}^{-(k+1)} e^{-\frac{\lambda_{t}^{2}}{2 h_{t}^{2}}-\frac{\delta_{t}}{h_{t}}}
\end{aligned}
$$

Thus, the conditional log-likelihood function up to a constant is

$$
l(\boldsymbol{\theta} ; k)=\sum_{t=1}^{T}\left\{k \log \left(\delta_{t}\right)-\log \Gamma(k)-(k+1) \log \left(h_{t}\right)-\frac{\lambda_{t}^{2}}{2 h_{t}^{2}}-\frac{\delta_{t}}{h_{t}}\right\} \equiv \sum_{t=1}^{T} l_{t}(\theta ; k),
$$

where $h_{t}=h_{t}(\theta)=\mu+\sum \alpha_{i}\left|\lambda_{t-i}\right|+\sum \beta_{i} \delta_{t-i}+\sum \gamma_{i} h_{t-i}$. Then, the joint maximum likelihood estimate of $\boldsymbol{\theta}$ and $k$ is defined as

$$
\left[\hat{\boldsymbol{\theta}}_{T}, \hat{k}_{T}\right]=\arg \max _{\boldsymbol{\theta}, k}\{l(\boldsymbol{\theta}, k)\}
$$

and it can be computed by the scoring algorithm

$$
\left[\boldsymbol{\theta}^{(m+1)}, k^{(m+1)}\right]^{\prime}=\left[\boldsymbol{\theta}^{(m)}, k^{(m)}\right]^{\prime}-\left[\nabla^{2} l\left(\boldsymbol{\theta}^{(m)}, k^{(m)}\right)\right]^{-1} \nabla l\left(\boldsymbol{\theta}^{(m)}, k^{(m)}\right),
$$

where $\nabla$ is the gradient with respect to $\boldsymbol{\theta}$ and $k$. Under the condition of strict stationarity and ergodicity, and some moment requirement for $\left\{h_{t}\right\},\left[\hat{\boldsymbol{\theta}}_{T}, \hat{k}_{T}\right]$ is consistent and asymptotically normal. We state the result in the following theorem. Details of the proof are referred to the Appendix.

Theorem 4. Assume the process $\left\{h_{t}\right\}$ is strictly stationary and ergodic, and $E\left(h_{t}^{2}\right)<\infty$. Assume in addition that the parameter space $\Theta \times \mathcal{K}$ is compact, with the maximum likelihood estimator defined in (39). Then

(i) $\hat{\boldsymbol{\theta}}_{T} \stackrel{\mathcal{P}}{\rightarrow} \boldsymbol{\theta}_{0}, \hat{k}_{T} \stackrel{\mathcal{P}}{\rightarrow} k_{0}$, as $T \rightarrow \infty$, where $k_{0}$ and $\boldsymbol{\theta}_{0}$ are the true parameters in population;

(ii) $T^{\frac{1}{2}}\left[\begin{array}{c}\hat{\boldsymbol{\theta}}_{T}-\boldsymbol{\theta}_{0} \\ \hat{k}_{T}-k_{0}\end{array}\right] \stackrel{\mathscr{D}}{\rightarrow} N\left(\mathbf{0}, \boldsymbol{I}^{-1}\left(\boldsymbol{\theta}_{0}, k_{0}\right)\right)$, where $\boldsymbol{I}\left(\boldsymbol{\theta}_{0}, k_{0}\right)=-E\left[\nabla^{2} l_{t}\left(\boldsymbol{\theta}_{0}, k_{0}\right)\right]$ is the Fisher information matrix evaluated at $\left(\boldsymbol{\theta}_{0}, k_{0}\right)$.

As an immediate consequence of the theorem, the asymptotic covariance matrix of $\left[\hat{\boldsymbol{\theta}}_{T}, \hat{k}_{T}\right]$ is $\frac{1}{T} \boldsymbol{I}^{-1}\left(\boldsymbol{\theta}_{0}, k_{0}\right)$. It can be consistently estimated by the inverse Hessian $-\left[\nabla^{2} l\left(\hat{\boldsymbol{\theta}}_{T}, \hat{k}_{T}\right)\right]^{-1}$, which is easily obtained from the scoring algorithm. Especially for Int-GARCH $(1,1,1)$, the condition for strict stationarity and ergodicity is the same as that for mean stationarity, that is, $E\left(x_{t}\right)<\infty$, and consequently $E\left(h_{t}\right)<\infty$ (see Lemma 3 in the Appendix). If we further assume 
covariance stationarity, i.e., $E\left(x_{t}^{2}\right)<\infty$ in view of Corollary 3, the joint maximum likelihood estimator $\left[\hat{\boldsymbol{\theta}}_{T}, \hat{k}_{T}\right]$ is consistent and asymptotically normal. We summarize the conclusion in the following corollary.

Corollary 5. Consider the Int-GARCH(1,1,1) model. If $E\left(x_{t}^{2}\right)<\infty$, then $\left[\hat{\boldsymbol{\theta}}_{T}, \hat{k}_{T}\right]$ satisfies both consistency and asymptotic normality.

Computation of the maximum likelihood estimate requires initial values of $k$ and $\boldsymbol{\theta}$, as well as starting values $\left\{h_{0}, \cdots, h_{-(m-1)}\right\}$ and $\left\{r_{0}, \cdots, r_{-(m-1)}\right\}$. The error distribution parameter $k$ can be conveniently initiated by the method of moments. Notice that

$$
\begin{aligned}
& E\left(\delta_{t}\right)=E\left(h_{t} \eta_{t}\right)=E\left(h_{t}\right) E\left(\eta_{t}\right)=k E\left(h_{t}\right), \\
& E\left|\lambda_{t}\right|=E\left|h_{t} \varepsilon_{t}\right|=E\left(h_{t}\right) E\left|\varepsilon_{t}\right|=\sqrt{2 / \pi} E\left(h_{t}\right),
\end{aligned}
$$

and consequently, $k=\sqrt{2 / \pi} \frac{E\left(\delta_{t}\right)}{E\left(\left|\lambda_{t}\right|\right)}$. Replacing $E\left(\delta_{t}\right)$ and $E\left(\left|\lambda_{t}\right|\right)$ by their sample estimates, we obtain the moment estimator of $k$ as

$$
k^{(0)}=\sqrt{2 / \pi} \frac{\overline{\delta_{t}}}{\overline{\left|\lambda_{t}\right|}}
$$

As for initial value of $\boldsymbol{\theta}$, recall from Theorem 1 that

$$
E\left(h_{t}\right)=\frac{\mu}{1-\sum_{i=1}^{m} \mu_{i}}=\frac{\mu}{1-\sqrt{\pi / 2} \sum_{i=1}^{p} \alpha_{i}-k \sum_{i=1}^{q} \beta_{i}-\sum_{i=1}^{w} \gamma_{i}} .
$$

So $\mu$ can be initialized by replacing $E\left(h_{t}\right)$ in (43) by its sample mean $\overline{h_{t}}$ and a rough guessing of $1-\sqrt{\pi / 2} \sum_{i=1}^{p} \alpha_{i}-k \sum_{i=1}^{q} \beta_{i}-\sum_{i=1}^{w} \gamma_{i}$, for example, 0.4. Additionally, initial values of $\alpha_{i}$ 's, $\beta_{i}$ 's, and $\gamma_{i}$ 's can be obtained by setting each of $\sqrt{\pi / 2} \sum_{i=1}^{p} \alpha_{i}, k \sum_{i=1}^{q} \beta_{i}$, and $\sum_{i=1}^{w} \gamma_{i}$ to be a small value, e.g., 0.2 , and by letting $\alpha_{1}=\cdots=\alpha_{p}, \beta_{1}=\cdots=\beta_{q}, \gamma_{1}=\cdots=\gamma_{w}$. If convergence is not achieved, a least squares estimation can be performed to obtain a more precise initial value for $\boldsymbol{\theta}$. Finally, we assume that the process $\left\{r_{t}\right\}$ starts from its infinite past with a finite mean and variance, so it is reasonable to let $h_{t}=E\left(h_{t}\right), t=0, \cdots,-(m-1)$. An alternative is to let $h_{t}=0, t=0, \cdots,-(m-1)$, assuming $\left\{r_{t}\right\}$ starts from constant intervals $\left\{r_{0}, \cdots, r_{-(m-1)}\right\}$. In either case, we let $r_{t}=E\left(r_{t}\right), t=0, \cdots,-(m-1)$.

Remark 4. In an earlier version of the paper, we proposed a two-stage estimation of the model parameters, which estimates $k$ by the method of moments and then computes the maximum likelihood estimate of $\boldsymbol{\theta}$ conditioning on $k$. It is slightly less efficient than the joint MLE, but it is computationally easier and converges to the limiting normal distribution faster, and therefore can be a good alternative in the situation of limited computational resources. It also provides a good initial value for running the full iteration of the joint MLE. Readers are referred to Supplementary Appendix B (online) for details.

\subsection{Simulation and finite sample performances}

Our simulation study considers four distinct Int-GARCH models with specific sets of parameter values. We are particularly interested in the Int-ARCH model, since very often in the real data 
analysis $\gamma_{1}$ is estimated to be 0 . So, after examining two full Int-GARCH models (Models I and II), we also consider two Int-ARCH models with $\gamma_{1}$ set to 0 (Models III and IV). The parameter values for the two Int-GARCH models are generated as follows.

$$
\begin{array}{ll}
\text { Models I and II }: & k \sim \operatorname{Unif}(1,2), \quad \mu \sim \operatorname{Unif}(0,0.1), \\
& \alpha_{1} \sim \operatorname{Unif}(0,0.2), \quad \beta_{1} \sim \operatorname{Unif}(0.1,0.6), \quad \gamma_{1} \sim \operatorname{Unif}(0,0.2) .
\end{array}
$$

For Int-ARCH models, $\beta_{1}$ is generally larger due to the removal of $\gamma_{1}$, so we generate the parameters for the two Int-ARCH models with $k, \mu, \alpha_{1}$ being the same as the Int-GARCH models but $\beta_{1}$ slightly larger.

$$
\begin{array}{ll}
\text { Models III and IV }: & k \sim \operatorname{Unif}(1,2), \quad \mu \sim \operatorname{Unif}(0,0.1), \\
& \alpha_{1} \sim \operatorname{Unif}(0,0.2), \quad \beta_{1} \sim \operatorname{Unif}(0.3,0.6) .
\end{array}
$$

All of the generations are subject to the constraint that each combination will result in a weakly stationary Int- $(\mathrm{G}) \mathrm{ARCH}$ process that achieves consistency and asymptotic normality for the maximum likelihood estimators. The exact parameter values are listed in Table 1. Realizations of the designed models are simulated using the initial values $h_{0}=0$ and $r_{0}=E\left(r_{t}\right)$. Plots of two simulated data sets are shown in Figure 2. Denote

\section{Figure 2 is approximately here.}

$$
\begin{aligned}
\gamma(s) & =\operatorname{Cov}\left(r_{t}, r_{t+s}\right), \\
\gamma_{\lambda}(s) & =\operatorname{Cov}\left(\lambda_{t}, \lambda_{t+s}\right), \\
\gamma_{\delta}(s) & =\operatorname{Cov}\left(\delta_{t}, \delta_{t+s}\right) .
\end{aligned}
$$

Recall that the theoretical ACF of $\left\{r_{t}\right\}$ is

$$
\rho(s)=\frac{\gamma(s)}{\gamma(0)}=\frac{\gamma_{\lambda}(s)+\gamma_{\delta}(s)}{\gamma_{\lambda}(0)+\gamma_{\delta}(0)}
$$

We consequently define the sample ACF of $\left\{r_{t}\right\}$ as

$$
\hat{\rho}(s)=\frac{\hat{\gamma_{\lambda}}(s)+\hat{\gamma_{\delta}}(s)}{\hat{\gamma_{\lambda}}(0)+\hat{\gamma_{\delta}}(0)},
$$

where $\hat{\gamma}_{\lambda}(s)$ and $\hat{\gamma}_{\delta}(s)$ are the sample auto-covariance functions of $\left\{\lambda_{t}\right\}$ and $\left\{\delta_{t}\right\}$, respectively. Figures 3 and 4 show the sample ACF's for simulated data sets with 1000 observations from an Int-GARCH(1,1,1) (Model I) and an Int-ARCH(1,1) (Model III), respectively.

Figure 3 is approximately here.

Figure 4 is approximately here.

For each of the four models, we simulate a data set with 2000 observations and estimate 
the parameters using the proposed maximum likelihood estimation. The process of simulation and estimation is repeated for 200 times independently, and the average results are reported in Table 1 . We see that, with a moderate sample of size 2000, the maximum likelihood estimates for $k$ and $\boldsymbol{\theta}$ are very close to the true values with small mean absolute errors. In addition, the asymptotic standard errors based on the asymptotic normality are compared to the empirical standard errors (i.e., the root mean squared errors), and they match very well.

\section{Empirical application}

In this section, we apply our Int-GARCH model to analyze 10 typical stocks and 4 indices data. The symbols of these stocks and indices are listed, for example, in Table 2. We obtained from Thomson Reuters (https://www.thomsonreuters.com/en.html) both the 5-minute intraday data and the daily-closing data from January 3, 2006 to December 30, 2011. As an example for demonstration purpose, the sample ACF of the BA (Bank of America) return range is displayed in Figure 5. It is very similar to the theoretical ACF of model I in Figure 1 from Section 5.2, which indicates the feasibility of our Int-GARCH model.

\section{Figure 5 is approximately here.}

\subsection{Data cleaning and ranking metrics}

In order to compare the empirical (in-sample and out-of-sample) performances of our IntGARCH model and the GARCH model, we use realized variance (RV) as the proxy for the "true" market variance. The RV using our 5-minute intraday data is computed as:

$$
\mathrm{RV}_{t}=\sum_{s}\left[y_{t}(s)-y_{t}(s-1)\right]^{2}
$$

As before, $y_{t}(s)$ denotes the log price of an asset at time $s$ on day $t$, and $y_{t}(s-1)$ denotes the log price of the previous "snapshot" which was taken 5 minutes ago.

Following Shephard and Sheppard (2010), we applied 4 filtration rules to clean the data. 1) When multiple quotes have the same timestamp, we replace all these with a single entry with the median bid and median ask price. 2) Delete entries for which the spread is negative. 3) Delete entries for which the spread is more than 50 times the median spread on that day. 4) Delete entries for which the mid-quote deviated by more than 10 mean absolute deviations from a rolling median centred but excluding the observation under consideration of 50 observations (i.e. 25 observations before and 25 after).

Denote the RV by $v_{t}$ and estimated volatility by $\hat{\sigma}_{t}^{2}$. It is shown in Meddahi (2001) that the ranking of volatility forecasts based on the $R^{2}$ from the Mincer-Zarnowitz regression

$$
v_{t}=\beta_{0}+\beta_{1} \hat{\sigma}_{t}^{2}+\epsilon_{t}
$$

is robust to noises in $v_{t}$. It is shown in Patton (2011) that the ranking using the negative quasi-likelihood (QLIKE) as the loss function is also robust to noises in the volatility proxy. 
Additionally, the heteroskedasticity-adjusted MSE (HMSE), although not robust to noises, is a popularly used loss function to compare volatility models. The definitions for the QLIKE and HMSE loss functions are given as:

$$
\begin{aligned}
\text { QLIKE } & =\frac{1}{N} \sum_{t=1}^{N}\left[\log \left(\hat{\sigma}_{t}^{2}\right)+\frac{v_{t}^{2}}{\hat{\sigma}_{t}^{2}}\right] \\
\mathrm{HMSE} & =\frac{1}{N} \sum_{t=1}^{N}\left(\frac{v_{t}^{2}}{\hat{\sigma}_{t}^{2}}-1\right),
\end{aligned}
$$

where $N$ is the length of sampling period.

\subsection{In-sample and out-of-sample results}

First, we compare the in-sample volatility estimation with that of $\operatorname{GARCH}(1,1)$. For this purpose, we fit an Int-GARCH model to the entire data from January 3, 2006 to December 30, 2011. The parameters are estimated by the maximum likelihood estimation we proposed in Section 5 and results are listed in Table 2. Occasionally, the estimate of the full model contains negative values. This means that the maximum of the likelihood function subject to the nonnegative constraints happens on the boundary, i.e., at least one of the estimates is 0 . This is equivalent to removing the corresponding parameter(s) and running the unconstrained estimation for the sub-model (e.g. Waterman, 1974). For such cases, the removed parameters are displayed as 0 exactly without a standard error. In general, the fitted models show that $\alpha_{1}$ is much smaller than $\beta_{1}$ both in magnitude and in statistical significance, indicating that, consistent to our expectation, the return range is of much more importance than a single "snapshot" return, in terms of their contributions to the volatility. Additionally, $\gamma_{1}$ also tends to be small, so most likely an Int-ARCH model is sufficient. The comparisons of $\operatorname{Int}-\operatorname{GARCH}(1,1,1)$ and $\operatorname{GARCH}(1,1)$ using all the three criteria ( $R^{2}$, QLIKE and HMSE) are shown in Table 3, which generally favor Int-GARCH, except for a few exceptions (i.e., $R^{2}$ of BAC, HMSE of MSFT).

Next, to examine the out-of-sample performance, we use the first 5 years (2006-2010) as training period and leave the last year (2011) for out-of-sample predictions. We consider 3 forecasting horizons: 1-step, 2-step, and 5-step, corresponding to one day, two day, and one week ahead predictions, respectively. The model parameters are updated daily and the h-stepahead predictions $(\mathrm{h}=1,2,5)$ are calculated according to equations (22)-(23). The predicts are compared to those from $\operatorname{GARCH}(1,1)$ model for the entire year of 2011, and the average results are shown in Tables 4, 5, and 6 , for $\mathrm{h}=1,2,5$, respectively. Similar to the in-sample comparison, the out-of-sample comparisons in Tables 4, 5, and 6 indicate the $\operatorname{Int-GARCH}(1,1,1)$ performs better than $\operatorname{GARCH}(1,1)$ for 1-step, 2-step, and 5-step ahead predictions, for most of the stocks (indices) and ranking metrics.

Finally, we also notice that occasionally the GARCH performs better than the Int-GARCH model in our experiments. For example, in Table $3, \mathrm{R}^{2}$ of $\mathrm{GARCH}$ is higher for BAC, and HMSE of GARCH is smaller for MSFT. In Table 6, the Int-GARCH is dominated by GARCH for QLIKE. Theoretically, the comparison between the GARCH and the Int-GARCH are complicated. Intuitively understanding, the advantage of our Int-GARCH model is that it makes 
full use of all possible values in the return range as volatility proxies and summarizes the information by integrating the GARCH mechanism with the random set theory. By comparison, the traditional GARCH model only considers use of the information of a single (closing-to-closing) return as the volatility proxy. Generally, the "summary" that accounts for more information from the data is expected to reflect the volatility more accurately. However, noting that different measures of model performance characterize different features of the model fitting the data, it is therefore possible occasionally that a simpler GARCH model with the point-valued return may outperform the more involved "summary" of Int-GARCH in terms of one performance measure. With regard to this, more extensive experiments with much more and larger data sets are needed to thoroughly investigate and optimize the performance of the Int-GARCH model in the near future.

\section{Conclusion}

In this paper, we have developed an interval-valued GARCH model for analyzing the return interval processes. It can be viewed as an extension of the point-valued GARCH model that allows for interval-valued returns to produce "information-richer" estimation of the volatility. Inferences of our Int-GARCH model can be made based on the maximum likelihood method, which has been shown to have both consistency and asymptotic normality. Our empirical study of stocks and indices data has demonstrated the advantages of Int-GARCH model over the GARCH for both in-sample and out-of-sample performances.

The Int-GARCH model forms a pioneer study in the area of conditional heteroscedasticity for interval-valued time series. Further improvements to the model can be made by incorporating interactions between the level and the range. Körner and Näther (2001) proposed for a more general space a generalized $L_{2}$ metric, which when restricted to $\mathcal{K}_{\mathcal{C}}(\mathbb{R})$ is

$$
\rho_{K}^{2}(x, y)=\sum_{(u, v) \in S^{0} \times S^{0}}\left(s_{x}(u)-s_{y}(u)\right)\left(s_{x}(v)-s_{y}(v)\right) K(u, v), \quad x, y \in \mathcal{K}_{\mathcal{C}}(\mathbb{R})
$$

where $K$ is a symmetric positive definite kernel. It can be represented by the center-radius form as

$$
\rho_{K}^{2}(x, y)=A_{11}\left(x^{C}-y^{C}\right)^{2}+A_{22}\left(x^{R}-y^{R}\right)^{2}+2 A_{12}\left(x^{C}-y^{C}\right)\left(x^{R}-y^{R}\right),
$$

where $A$ as a function of $K$ is a symmetric positive definite matrix. So $\rho_{K}$ is a further generalization of $\rho_{W}$ in (19) that takes into account the correlation between the center and the radius, and can be utilized to model the level-range interaction.

Supplementary Appendix A Proofs of corollaries and lemmas.

Supplementary Appendix B The two-stage estimation of $k$ and $\boldsymbol{\theta}$.

Data Availability Statement The data that support the findings of this study are provided as online supporting information. 


\section{Acknowledgment}

The authors would like to thank the editor and two anonymous referees for carefully reading the manuscript. Their valuable comments and suggestions have led to a much better presentation of the paper.

\section{References}

Arroyo J, Espińola R, Maté. C. 2011. Different approaches to forecast interval time series: a comparison in finance. Computational Economics 37: 169-191.

Artstein Z, Vitale RA. 1975. A strong law of large numbers for random compact sets. Annals of Probability 5: 879-882.

Aumann RJ. 1965. Integrals of set-valued functions. Journal of Mathematical Analysis and Applications 12: 1-12.

Basawa IV, Feigin PD, Heyde CC. 1976. Asymptotic properties of maximum likelihood estimators for stochastic processes. Sankhya 38, Series A: 259-270.

Billard L, Diday E. 2003. From the statistics of data to the statistics of knowledge: symbolic data analysis. Journal of the American Statistical Association 98: 470-487.

Billard L. 2007. Dependencies and variation components of symbolic interval-valued data. In: Selected Contributions in Data Analysis and Classification: 3-12. Springer, Berlin Heidelberg.

Blanco-Fernández A, Corral N, González-Rodríguez G. 2011. Estimation of a flexible simple linear model for interval data based on set arithmetic. Computational Statistics and Data Analysis 55: 2568-2578.

Bollerslev T. 1986. Generalized autoregressive conditional heteroskedasticity. Journal of Econometrics 31: 307-327.

Debreu G. 1967. Integration of Correspondences. In: Proceedings of the Fifth Berkeley Symposium on Mathematical Statistics and Probability II: 351-372. Berkeley, California.

D'Esposito MR, Palumbo F, Ragozini, G. 2012. Interval Archetypes: a new tool for interval data analysis. Statistical Analysis \& Data Mining 5: 322-335.

Diamond P. 1990. Least squares fitting of compact set-valued data. Journal of Mathematical Analysis and Applications 147: 531-544.

Ding Z, Granger CWJ, Engle RF. 1993. A long memory property of stock market returns and a new model. Journal of Empirical Finance 1: 83-106.

Engle RF. 1982. Autoregressive conditional heteroskedasticity with estimates of the variance of U.K. inflation. Econometrica 50: 987-1008. 
Fischer H, Blanco-Fernández A, Winker P. 2016. Predicting stock return volatility: Can we benefit from regression models for return intervals? Journal of Forecasting 35: 113-146.

Fréchet M. 1948. Les éléments aléatoires de natures quelconque dans un espace distancié. Ann. Inst. H. Poincaré 10: 215-310.

Gil MA, Lopez MT, Lubiano MA, Montenegro M. 2001. Regression and correlation analyses of a linear relation between random intervals. Test 10(1): 183-201.

Gil MA, Lubiano MA, Montenegro M, Lopez MT. 2002. Least squares fitting of an affine function and strength of association for interval-valued data. Metrika 56: 97-111.

Gil MA, González-Rodríguez G, Colubi A, Montenegro, M. 2007. Testing linear independence in linear models with interval-valued data. Computational Statistics \& Data Analysis 51: 3002-3015.

Gioia F, Lauro CN. 2006. Principal component analysis on interval data. Computational Statistics 21: 343-363.

González-Rodríguez G, Blanco A, Corral N, Colubi A. 2007. Least squares estimation of linear regression models for convex compact random sets. Advances in Data Analysis and Classification 1: 67-81.

Han A, Hong Y, Lai K, Wang S. 2008. Interval time series analysis with an application to the sterling-dollar exchange rate. Journal of Systems Science and Complexity 21(4): 550-565.

Han A, Hong Y, Wang S. 2012. Autoregressive conditional models for interval-valued time series data. Preprint.

He LT, Hu C. 2009. Impacts of interval computing on stock market variability forecasting. Computational Economics 33(3): 263-276.

Hörmander L. 1954. Sur la fonction d'appui des ensembles convexes dans un espace localement convexe. Arkiv för Mat. 3: 181-186.

Hu C, He L. 2007. An application of interval methods to stock market forecasting. Reliable Computing 13(5): 423-434.

Hiai F, Umegaki, H. 1977. Integrals, conditional expectations and martingales of multivalued functions. Journal of Multivariate Analysis 7: 149-182.

Kendall DG. 1974. Foundations of a theory of random sets. In: Stochastic Geometry (eds. Harding EF, Kendall DG). John Wiley \& Sons, New York.

Körner R. 1995. A variance of compact convex random sets. Preprint. Institut für Stochastik, Bernhard-von-Cotta-Str. 2-09599 Freiberg.

Körner R. 1997. On the variance of fuzzy random variables. Fuzzy Sets and Systems 92: 83-93. 
Körner R, Näther W. 1998. Linear regression with random fuzzy variables: extended classical estimates, best linear estimates, least squares estimates. Information Sciences 109: 95-118.

Körner R, Näther W. 2001. On the variance of random fuzzy variables. In: Statistical Modeling, Analysis and Management of Fuzzy Data (eds. Bertoluzza C, Gil M, Ralescu, D): 25-42. Physica-Verlag, Heidelberg.

Lyashenko NN. 1982. Limit theorem for sums of independent compact random subsets of Euclidean space. Journal of Soviet Mathematics 20: 2187-2196.

Maia ALS, Francisco FAT, Ludermir TB. 2008. Forecasting models for interval-valued time series. Neurocomputing 71: 3344-3352.

Matheron G. 1975. Random Sets and Integral Geometry. John Wiley \& Sons, New York.

Meddahi N. 2001. A theoretical comparison between integrated and realized volatilities. Preprint. Université de Montréal.

Molchanov I. 2005. Theory of Random Sets. Springer-Verlag, London.

Montenegro M, Casals MR, Colubi A, Gil MA. 2008. Testing 'Two-Sided' hypothesis about the mean of an nterval-valued random set. In: Soft Methods for Handling Variability and Imprecision (eds. Dubois D, Lubiano MA, Prade H, Gil MA, Grzegorzewski P, Hryniewicz O) 48: 133-139. Springer-Verlag, Berlin, Heidelberg.

Näther W, Wünsche A. 2007. On the conditional variance of fuzzy random variables. Metrika 65: 109-122.

Lima Neto EA, Carvalho FAT. 2008. Centre and range method for fitting a linear regression model to symbolic interval data. Computational Statistics \& Data Analysis 52: 1500-1515.

Lima Neto EA, Carvalho FAT. 2010. Constrained linear regression models for symbolic intervalvalued variables. Computational Statistics \& Data Analysis 54: 333-347.

Lima Neto EA, Anjos UU. 2015. Regression model for interval-valued variables based on copulas. Journal of Applied Statistics 42(9): 2010-2029.

Patton AJ. 2011. Volatility forecast comparison using imperfect volatility proxies. Journal of Econometrics 160: 246-256.

Ràdström, H. 1952. An embedding theorem for spaces of convex sets. Proc. Amer. Math. Soc. 3: $165-169$.

Schwert W. 1990. Stock volatility and the rash of '87. Review of Financial Studies 3(1): 77-102.

Shephard N, Sheppard K. 2010. Realizing the future: forecasting with high-frequency-based volatility (HEAVY) models. Journal of Applied Econometrics 25: 197-231.

Sun Y, Ralescu D. 2015. A Normal hierarchical model and minimum contrast estimation for random intervals. Annals of the Institute of Statistical Mathematics 67: 313-333. 
Sun Y. 2017. Asymptotic tests for interval-valued means. Statistics and Probability Letters 121: 70-77.

Taylor S. 1986. Modeling Financial Time Series. John Wiley \& Sons, New York.

Waterman MS. 1974. A restricted least squares problem. Technometrics 16(1): 135-136.

Wei Y, Wang S, Wang H. 2017. Interval-valued data regression using partial linear model. Journal of Statistical Computation and Simulation 87(16): 3175-3194.

Weiss AA. 1986. Asymptotic theory for ARCH models: estimation and testing. Econometric Theory 2(1): 107-131.

Yang W, Han A, Wang S. 2014. Forecasting financial volatility with interval-valued time series data. Vulnerability, Uncertainty, and Risk: 1224-1233.

\section{Appendix}

\subsection{Proof of Theorem 1}

Proof. By the definition of (26), (16) can be rewritten as

$$
\begin{aligned}
h_{t} & =\mu+\sum_{i=1}^{p} \alpha_{i}\left|\epsilon_{t-i}\right| h_{t-i}+\sum_{i=1}^{q} \beta_{i} \eta_{t-i} h_{t-i}+\sum_{i=1}^{w} \gamma_{i} h_{t-i} \\
& =\mu+\sum_{i=1}^{m} x_{i, t-i} h_{t-i} .
\end{aligned}
$$

Expanding $h_{t}$ recursively, we obtain

$$
\begin{aligned}
h_{t}= & \mu+\sum_{i=1}^{m} x_{i, t-i}\left(\mu+\sum_{j=1}^{m} x_{j, t-i-j} h_{t-i-j}\right) \\
= & \mu\left(1+\sum_{i=1}^{m} x_{i, t-i}\right)+\sum_{i=1}^{m} \sum_{j=1}^{m} x_{i, t-i} x_{j, t-i-j} h_{t-i-j} \\
= & \cdots \\
= & \mu\left[1+\sum_{n=1}^{N} \sum_{i_{1}=1}^{m} \cdots \sum_{i_{n}=1}^{m}\left(\prod_{j=1}^{n} x_{i_{j}, t-i_{1}-\cdots-i_{j}}\right)\right] \\
& +\sum_{i_{1}=1}^{m} \cdots \sum_{i_{N+1}=1}^{m}\left(\prod_{j=1}^{N+1} x_{i_{j}, t-i_{1}-\cdots-i_{j}}\right) h_{t-i_{1}-\cdots-i_{N+1}} .
\end{aligned}
$$


Notice that $x_{i, t}$ and $x_{j, s}$ are independent, $\forall i, j \in \mathbb{N}$ and $t \neq s$. Taking expectations on both sizes of (46), we get

$$
\begin{aligned}
E\left(h_{t}\right)= & \mu\left[1+\sum_{n=1}^{N} \sum_{i_{1}=1}^{m} \cdots \sum_{i_{n}=1}^{m}\left(\prod_{j=1}^{n} \mu_{i_{j}}\right)\right] \\
& +\sum_{i_{1}=1}^{m} \cdots \sum_{i_{N+1}=1}^{m}\left(\prod_{j=1}^{N+1} \mu_{i_{j}}\right) E\left(h_{t-i_{1}-\cdots-i_{N+1}}\right) \\
& :=I+I I .
\end{aligned}
$$

The first term

$$
I=\mu\left[1+\sum_{n=1}^{N} \sum_{i_{1}=1}^{m} \cdots \sum_{i_{n}=1}^{m}\left(\prod_{j=1}^{n} \mu_{i_{j}}\right)\right]=\mu\left[1+\sum_{n=1}^{N}\left(\sum_{j=1}^{m} \mu_{j}\right)^{n}\right], \quad \forall N \in \mathbb{N} .
$$

The second term

$$
\begin{aligned}
I I & =\sum_{i_{1}=1}^{m} \cdots \sum_{i_{N+1}=1}^{m}\left(\prod_{j=1}^{N+1} \mu_{i_{j}}\right) E\left(h_{t-i_{1}-\cdots-i_{N+1}}\right) \\
& \leq \sum_{i_{1}=1}^{m} \cdots \sum_{i_{N+1}=1}^{m}\left(\prod_{j=1}^{N+1} \mu_{i_{j}}\right) \max \left\{E\left(h_{t-l}\right): N+1 \leq l \leq k(N+1)\right\} \\
& =\max \left\{E\left(h_{t-l}\right): N+1 \leq l \leq k(N+1)\right\}\left(\sum_{j=1}^{m} \mu_{j}\right)^{N+1}, \forall N \in \mathbb{N} .
\end{aligned}
$$

Therefore, $E\left(h_{t}\right)<\infty$ if and only if $\sum_{i=1}^{m} \mu_{i}<1$. When it is satisfied,

$$
\begin{aligned}
E\left(h_{t}\right)= & \lim _{N \rightarrow \infty} \mu\left[1+\sum_{n=1}^{N}\left(\sum_{j=1}^{m} \mu_{j}\right)^{n}\right] \\
& +\lim _{N \rightarrow \infty} \max \left\{E\left(h_{t-l}\right): N+1 \leq l \leq k(N+1)\right\}\left(\sum_{j=1}^{m} \mu_{j}\right)^{N+1} \\
= & \frac{\mu}{1-\sum_{i=1}^{m} \mu_{i}},
\end{aligned}
$$

by the finiteness of $E\left(h_{-\infty}\right)$. The formula for $E\left(r_{t}\right)$ follows immediately from the Aumann expectation.

\subsection{Proof of Theorem 2}

Proof. Let $\tilde{H}_{t}=\left[h_{t}, h_{t-1}, \cdots, h_{t-m+1}\right]^{\prime}$ with $m=\max \{p, q, w\}$. It follows from (16) together with (31) that

$$
\tilde{H}_{t}=\mathbf{a}+A_{t} \tilde{H}_{t-1}
$$


where $\mathbf{a}=[\mu, 0, \cdots, 0]^{\prime}$ is an $m$-dimensional vector. Then under the mean stationarity in Theorem 1, i.e., $\sum_{i=1}^{m} E\left(x_{i, t-i}\right)<1$, or equivalently, the eigenvalues of the matrix $E\left(A_{t}\right)$ being within a unit circle, we can express (47) as

$$
\tilde{H}_{t}=\mathbf{a}+\sum_{j=1}^{\infty} \prod_{k=1}^{j} A_{t-k+1} \mathbf{a}
$$

Notice that $A_{t}$ 's are independent across time $t$. Thus, with $\otimes$ standing for Kronecker product, it is easy to show from (48) that $E\left(h_{t}^{2}\right)<\infty$ is equivalent to $E\left(\tilde{H}_{t} \otimes \tilde{H}_{t}\right)$ being finite, which holds true if and only if

$$
E \sum_{j=1}^{\infty} \prod_{k=1}^{j}\left(A_{t-k+1} \otimes A_{t-k+1}\right)=\sum_{j=1}^{\infty}\left[E\left(A_{t} \otimes A_{t}\right)\right]^{j}
$$

converges, i.e., the eigenvalues of the matrix $E\left(A_{t} \otimes A_{t}\right)$ are within a unit circle. This completes the proof.

\subsection{Proof of Theorem 3}

Proof. Notice, $\forall t, s \in \mathbb{N}$,

$$
\operatorname{Cov}\left(r_{t}, r_{t+s}\right)=\operatorname{Cov}\left(h_{t} \varepsilon_{t}, h_{t+s} \varepsilon_{t+s}\right)+\operatorname{Cov}\left(h_{t} \eta_{t}, h_{t+s} \eta_{t+s}\right)
$$

Since $\left\{\varepsilon_{t}\right\}$ are i.i.d., the first term is seen to be

$$
\begin{aligned}
\operatorname{Cov}\left(h_{t} \varepsilon_{t}, h_{t+s} \varepsilon_{t+s}\right) & =E\left[\left(h_{t} \varepsilon_{t}-E\left(h_{t} \varepsilon_{t}\right)\right)\left(h_{t+s} \varepsilon_{t+s}-E\left(h_{t+s} \varepsilon_{t+s}\right)\right)\right] \\
& =E\left(h_{t} h_{t+s} \varepsilon_{t} \cdot \varepsilon_{t+s}\right) \\
& = \begin{cases}E\left(h_{t}^{2}\right), & s=0 \\
0, & |s|>0 .\end{cases}
\end{aligned}
$$

Similarly, the second term becomes

$$
\begin{aligned}
\operatorname{Cov}\left(h_{t} \eta_{t}, h_{t+s} \eta_{t+s}\right) & =E\left[\left(h_{t} \eta_{t}-k E\left(h_{t}\right)\right)\left(h_{t+s} \eta_{t+s}-k E\left(h_{t+s}\right)\right)\right] \\
& =E\left(h_{t} h_{t+s} \eta_{t} \eta_{t+s}\right)-k^{2} E\left(h_{t}\right) E\left(h_{t+s}\right) \\
& = \begin{cases}\left(k+k^{2}\right) E\left(h_{t}^{2}\right)-k^{2}\left[E\left(h_{t}\right)\right]^{2}, & s=0 \\
k E\left(h_{t} h_{t+s} \eta_{t}\right)-k^{2}\left[E\left(h_{t}\right)\right]^{2}, & |s|>0 .\end{cases}
\end{aligned}
$$

Plugging (50) and (51) into (49), we obtain

$$
\operatorname{Cov}\left(r_{t}, r_{t+s}\right)= \begin{cases}\left(1+k+k^{2}\right) E\left(h_{t}^{2}\right)-k^{2}\left[E\left(h_{t}\right)\right]^{2}, & s=0 \\ k E\left(h_{t} h_{t+s} \eta_{t}\right)-k^{2}\left[E\left(h_{t}\right)\right]^{2}, & |s|>0\end{cases}
$$


where $E\left(h_{t}\right)=\frac{\mu}{1-C_{1}}, E\left(h_{t}^{2}\right)=\mu^{2} \frac{C_{1}+1}{\left(C_{2}-1\right)\left(C_{1}-1\right)}$, and

$$
E\left(h_{t} h_{t+s} \eta_{t}\right)=\frac{\mu^{2} k}{C_{1}-1}\left(-\frac{C_{1}^{s}-1}{C_{1}-1}+\frac{C_{1}^{s}+C_{1}^{s-1}}{C_{2}-1} \cdot\left[\alpha_{1} \sqrt{\frac{2}{\pi}}+\beta_{1}(1+k)+\gamma_{1}\right]\right) .
$$

(see Lemma 1, Section 2 of Supplementary Appendix A).

\subsection{Proof of Theorem 4}

Proof. Let $l_{t}(\boldsymbol{\theta}, k)=k \log \left(\delta_{t}\right)-\log \Gamma(k)-(k+1) \log \left(h_{t}\right)-\frac{\lambda_{t}^{2}}{2 h_{t}^{2}}-\frac{\delta_{t}}{h_{t}}$, that is, $l(\boldsymbol{\theta}, k)=\sum_{t=1}^{T} l_{t}(\boldsymbol{\theta}, k)$.

The elements in $\nabla l$ are calculated to be

$$
\begin{aligned}
\frac{\partial l}{\partial k} & =\sum_{t} \frac{\partial l_{t}}{\partial k}=\sum_{t}\left[\log \left(\delta_{t}\right)-\frac{\Gamma^{\prime}(k)}{\Gamma(k)}-\log \left(h_{t}\right)\right]=\sum_{t}\left[\log \left(\frac{\delta_{t}}{h_{t}}\right)-\frac{\Gamma^{\prime}(k)}{\Gamma(k)}\right], \\
\frac{\partial l}{\partial \mu} & =\sum_{t} \frac{\partial l_{t}}{\partial h_{t}}=\sum_{t}\left(-\frac{k+1}{h_{t}}+\frac{\lambda_{t}^{2}}{h_{t}^{3}}+\frac{\delta_{t}}{h_{t}^{2}}\right), \\
\frac{\partial l}{\partial \alpha_{i}} & =\sum_{t} \frac{\partial l_{t}}{\partial h_{t}}\left|\lambda_{t-i}\right|, \quad \frac{\partial l}{\partial \beta_{i}}=\sum_{t} \frac{\partial l_{t}}{\partial h_{t}} \delta_{t-i}, \quad \frac{\partial l}{\partial \gamma_{i}}=\sum_{t} \frac{\partial l_{t}}{\partial h_{t}} h_{t-i} .
\end{aligned}
$$

In addition, the elements in $\nabla^{2} l_{t}$ are

$$
\frac{\partial^{2} l_{t}}{\partial k^{2}}, \quad \frac{\partial^{2} l_{t}}{\partial k \partial \boldsymbol{\theta}_{i}}, \quad \frac{\partial^{2} l_{t}}{\partial \boldsymbol{\theta}_{i} \partial \boldsymbol{\theta}_{j}}=\frac{\partial^{2} l_{t}}{\partial h_{t}^{2}} \frac{\partial h_{t}}{\partial \boldsymbol{\theta}_{i}} \frac{\partial h_{t}}{\partial \boldsymbol{\theta}_{j}} .
$$

(i) To prove consistency, we follow Weiss (1986) to verify the three conditions in Basawa et al.

(1976) that guarantee the existence of a consistent root of the gradient equation.

(1) $\frac{1}{T} \sum_{t} \nabla l_{t}\left(\boldsymbol{\theta}_{0}, k_{0}\right) \stackrel{\mathcal{P}}{\rightarrow} \mathbf{0}$ as $n \rightarrow \infty$;

(2) There exists a nonrandom positive definite matrix $M\left(\boldsymbol{\theta}_{0}, k_{0}\right)$ such that $\forall \epsilon>0$ :

$$
P\left(-\frac{1}{T} \sum_{t} \nabla^{2} l_{t}\left(\boldsymbol{\theta}_{0}, k_{0}\right) \geq M\left(\boldsymbol{\theta}_{0}, k_{0}\right)\right)>1-\epsilon, \quad \forall T>T_{1}(\epsilon)
$$

(3) There exists a constant $M<\infty$ such that

$$
E\left\|\nabla_{i j k}^{3} l_{t}(\boldsymbol{\theta}, k)\right\|<M, \quad \forall \boldsymbol{\theta} \in \Theta, k \in \mathcal{K} .
$$

For (1), notice that evaluated at $\boldsymbol{\theta}=\boldsymbol{\theta}_{0}$ and $k=k_{0}$,

$$
E\left(\frac{\partial l_{t}}{\partial h_{t}}\right)=E\left[E\left(\frac{\partial l_{t}}{\partial h_{t}} \mid \mathcal{F}_{t-1}\right)\right]=E\left(-\frac{k+1}{h_{t}}+\frac{h_{t}^{2}}{h_{t}^{3}}+\frac{k h_{t}}{h_{t}^{2}}\right)=0 .
$$


Thus,

$$
\begin{aligned}
& E\left(\frac{\partial l_{t}}{\partial \mu}\right)=E\left(\frac{\partial l_{t}}{\partial h_{t}}\right)=0, \\
& E\left(\frac{\partial l_{t}}{\partial \alpha_{i}}\right)=E\left(\frac{\partial l_{t}}{\partial h_{t}}\left|\lambda_{t-i}\right|\right)=E\left[\left|\lambda_{t-i}\right| E\left(\frac{\partial l_{t}}{\partial h_{t}} \mid \mathcal{F}_{t-1}\right)\right]=0, \\
& E\left(\frac{\partial l_{t}}{\partial \beta_{i}}\right)=E\left(\frac{\partial l_{t}}{\partial h_{t}} \delta_{t-i}\right)=E\left[\delta_{t-i} E\left(\frac{\partial l_{t}}{\partial h_{t}} \mid \mathcal{F}_{t-1}\right)\right]=0, \\
& E\left(\frac{\partial l_{t}}{\partial \gamma_{i}}\right)=E\left(\frac{\partial l_{t}}{\partial h_{t}} h_{t-i}\right)=E\left[h_{t-i} E\left(\frac{\partial l_{t}}{\partial h_{t}} \mid \mathcal{F}_{t-1}\right)\right]=0 .
\end{aligned}
$$

In addition, since $\eta_{t} \stackrel{i . i . d .}{\sim} \Gamma\left(k_{0}, 1\right)$

$$
E\left(\frac{\partial l_{t}}{\partial k}\left(\boldsymbol{\theta}_{0}, k_{0}\right)\right)=E\left[E\left(\frac{\partial l_{t}}{\partial k}\left(\boldsymbol{\theta}_{0}, k_{0}\right) \mid \mathcal{F}_{t-1}\right)\right]=E\left[\log \left(\eta_{t}\right)-\frac{\Gamma^{\prime}\left(k_{0}\right)}{\Gamma\left(k_{0}\right)}\right]=0 .
$$

Therefore, $E\left[\nabla l_{t}\left(\boldsymbol{\theta}_{0}, k_{0}\right)\right]=0$. By the ergodic theorem, $\frac{1}{T} \sum_{t=1}^{T} \nabla l_{t}\left(\boldsymbol{\theta}_{0}, k_{0}\right) \stackrel{\mathcal{P}}{\rightarrow} \mathbf{0}, T \rightarrow \infty$.

For (2), in view of the ergodic theorem, it is sufficient to verify $E\left[-\nabla^{2} l_{t}\left(\boldsymbol{\theta}_{0}, k_{0}\right)\right]<\infty$. Notice

$$
\frac{\partial^{2} l_{t}}{\partial h_{t}^{2}}=\frac{k+1}{h_{t}^{2}}-\frac{3 \lambda_{t}^{2}}{h_{t}^{4}}-\frac{2 \delta_{t}}{h_{t}^{3}} .
$$

Therefore,

$$
\begin{aligned}
E\left[\frac{\partial^{2} l_{t}}{\partial \boldsymbol{\theta}_{i} \partial \boldsymbol{\theta}_{j}}\left(\boldsymbol{\theta}_{0}, k_{0}\right)\right] & =E\left[E\left(\frac{\partial^{2} l_{t}}{\partial \boldsymbol{\theta}_{i} \partial \boldsymbol{\theta}_{j}}\left(\boldsymbol{\theta}_{0}, k_{0}\right)\right) \mid \mathcal{F}_{t-1}\right] \\
& =E\left[\left(\frac{k_{0}+1}{h_{t}^{2}}-\frac{3 h_{t}^{2}}{h_{t}^{4}}-\frac{2 k_{0} h_{t}}{h_{t}^{3}}\right) \frac{\partial h_{t}}{\partial \boldsymbol{\theta}_{i}} \frac{\partial h_{t}}{\partial \boldsymbol{\theta}_{j}}\left(\boldsymbol{\theta}_{0}\right)\right] \\
& =-\left(k_{0}+2\right) E\left[h_{t}^{-2} \frac{\partial h_{t}}{\partial \boldsymbol{\theta}_{i}} \frac{\partial h_{t}}{\partial \boldsymbol{\theta}_{j}}\left(\boldsymbol{\theta}_{0}\right)\right] .
\end{aligned}
$$

Since $h_{t}^{-1} \frac{\partial h_{t}}{\partial \boldsymbol{\theta}_{i}}\left(\boldsymbol{\theta}_{0}\right)$ is bounded by $\max \left\{\frac{1}{\alpha_{i}}, \frac{1}{\beta_{j}}, \frac{1}{\gamma_{k}}, \frac{1}{\mu}\right\}$, we have $E\left[-\frac{\partial^{2} l_{t}}{\partial \boldsymbol{\theta}_{i} \partial \boldsymbol{\theta}_{j}}\left(\boldsymbol{\theta}_{0}, k_{0}\right)\right]<\infty$. Similarly,

$$
E\left[\frac{\partial^{2} l_{t}}{\partial k \partial \boldsymbol{\theta}_{i}}\left(\boldsymbol{\theta}_{0}, k_{0}\right)\right]=E\left[-\frac{\partial}{\partial \boldsymbol{\theta}_{i}} \log \left(h_{t}\right)\left(\boldsymbol{\theta}_{0}\right)\right]=-E\left[h_{t}^{-1} \frac{\partial h_{t}}{\partial \boldsymbol{\theta}_{i}}\left(\boldsymbol{\theta}_{0}\right)\right]<\infty .
$$

In addition,

$$
\frac{\partial^{2} l_{t}}{\partial k^{2}}\left(\boldsymbol{\theta}_{0}, k_{0}\right)=\frac{\partial}{\partial k}\left[\log \left(\delta_{t}\right)-\frac{\Gamma^{\prime}(k)}{\Gamma(k)}-\log \left(h_{t}\right)\right]\left(k_{0}\right)=-\left[\frac{\Gamma^{\prime}(k)}{\Gamma(k)}\right]^{\prime}\left(k_{0}\right),
$$

so

$$
E\left[\frac{\partial^{2} l_{t}}{\partial k^{2}}\left(\boldsymbol{\theta}_{0}, k_{0}\right)\right]=-\left[\frac{\Gamma^{\prime}(k)}{\Gamma(k)}\right]^{\prime}\left(k_{0}\right)=-\phi^{(1)}\left(k_{0}\right)<\infty
$$

where $\phi(\cdot)$ is the polygamma function. Thus, $E\left[-\nabla^{2} l_{t}\left(\boldsymbol{\theta}_{0}, k_{0}\right)\right]<\infty$, and hence condition (2) is satisfied. 
For (3), we note that

$$
\begin{aligned}
\frac{\partial^{3} l_{t}}{\partial \boldsymbol{\theta}_{i} \partial \boldsymbol{\theta}_{j} \partial \boldsymbol{\theta}_{k}} & =\frac{\partial^{3} l_{t}}{\partial h_{t}^{3}} \frac{\partial h_{t}}{\partial \boldsymbol{\theta}_{i}} \frac{\partial h_{t}}{\partial \boldsymbol{\theta}_{j}} \frac{\partial h_{t}}{\partial \boldsymbol{\theta}_{k}} \\
& =-2\left[(k+1)-6 h_{t}^{-2} \lambda_{t}^{2}-3 h_{t}^{-1} \delta_{t}\right] h_{t}^{-3} \frac{\partial h_{t}}{\partial \boldsymbol{\theta}_{i}} \frac{\partial h_{t}}{\partial \boldsymbol{\theta}_{j}} \frac{\partial h_{t}}{\partial \boldsymbol{\theta}_{k}}
\end{aligned}
$$

By the compactness of $\Theta, h_{t}^{-1} \frac{\partial h_{t}}{\partial \boldsymbol{\theta}_{i}}$ is uniformly bounded, and so is $h_{t}^{-3} \frac{\partial h_{t}}{\partial \boldsymbol{\theta}_{i}} \frac{\partial h_{t}}{\partial \boldsymbol{\theta}_{j}} \frac{\partial h_{t}}{\partial \boldsymbol{\theta}_{k}}$. Similarly, $h_{t}^{-1}, h_{t}^{-2}$ are also uniformly bounded since $h_{t}>\mu$. So to show $\frac{\partial^{3} l_{t}}{\partial \boldsymbol{\theta}_{i} \partial \boldsymbol{\theta}_{j} \partial \boldsymbol{\theta}_{k}}$ has a uniformly bounded expectation, it suffices to have $E\left(\lambda_{t}^{2}\right)<\infty$, which is equivalent to $E\left(h_{t}^{2}\right)<\infty$ and guaranteed by the condition of weak stationarity. Similarly,

$$
\frac{\partial^{3} l_{t}}{\partial k \partial \theta_{i} \partial \theta_{j}}=\frac{1}{h_{t}^{2}} \frac{\partial h_{t}}{\partial \theta_{i}} \frac{\partial h_{t}}{\partial \theta_{j}}, \quad \frac{\partial^{3} l_{t}}{\partial k^{2} \partial \theta_{i}}=0, \quad \frac{\partial^{3} l_{t}}{\partial k^{3}}=-\phi^{(2)}(k)
$$

all have uniformly bounded expectations. Hence, condition (3) is satisfied under the assumption of weak stationarity.

(ii) The requirements in Basawa et al. (1976) for the asymptotic normality of the maximum likelihood estimator are:

(1) $\frac{1}{\sqrt{T}} \sum_{t} \nabla l_{t}\left(\boldsymbol{\theta}_{0}, k_{0}\right) \stackrel{\mathscr{D}}{\rightarrow} N(\mathbf{0}, B), T \rightarrow \infty$, for a nonrandom positive definite matrix $B$;

(2) $-\frac{1}{T} \sum_{t} \nabla^{2} l_{t}\left(\boldsymbol{\theta}_{0}, k_{0}\right) \stackrel{\mathcal{P}}{\rightarrow} \boldsymbol{I}\left(\boldsymbol{\theta}_{0}, k_{0}\right)=E\left[-\nabla^{2} l_{t}\left(\boldsymbol{\theta}_{0}, k_{0}\right)\right]=E\left[\nabla \nabla^{T} l_{t}\left(\boldsymbol{\theta}_{0}, k_{0}\right)\right], T \rightarrow \infty$, where $\boldsymbol{I}\left(\boldsymbol{\theta}, k_{0}\right)$ is the Fisher information matrix at $\boldsymbol{\theta}=\boldsymbol{\theta}_{0}$ and $k=k_{0}$;

(3) Condition (3) for consistency.

For $(1)$, since $E\left(\nabla l_{t}\left(\boldsymbol{\theta}_{0}, k_{0}\right) \mid \mathcal{F}_{t-1}\right)=0$, by the martingale central limit theorem,

$$
\frac{1}{\sqrt{T}} \sum_{t} \nabla l_{t}\left(\boldsymbol{\theta}_{0}, k_{0}\right) \stackrel{\mathscr{D}}{\rightarrow} N\left(\mathbf{0}, \boldsymbol{I}\left(\boldsymbol{\theta}, k_{0}\right)\right), \quad T \rightarrow \infty,
$$

Condition (2) is already shown by the ergodic theorem in the preceding argument. Hence, the proof is completed. 
Table 1: Average result of 200 repeated simulations from two Int-GARCH(1,1,1) models (I, II) and two Int-ARCH $(1,1)$ models (III, IV).

\begin{tabular}{|c|c|c|c|c|c|c|}
\hline \multirow{3}{*}{$\begin{array}{c}\text { Model } \\
\text { I }\end{array}$} & \multicolumn{2}{|c|}{ Parameters } & $\begin{array}{c}\text { Mean } \\
\text { Estimate }\end{array}$ & $\begin{array}{c}\text { Mean } \\
\text { Absolute Error }\end{array}$ & $\begin{array}{c}\text { Empirical } \\
\text { Standard Error }\end{array}$ & $\begin{array}{c}\text { Asymptotic } \\
\text { Standard Error }\end{array}$ \\
\hline & $k$ & 1.8147 & 1.8164 & 0.0283 & 0.0344 & 0.0327 \\
\hline & $\mu$ & 0.0906 & 0.0908 & 0.0046 & 0.0058 & 0.0062 \\
\hline & $\alpha_{1}$ & 0.0318 & 0.0325 & 0.0115 & 0.0146 & 0.0159 \\
\hline & $\beta_{1}$ & 0.3740 & 0.3739 & 0.0088 & 0.0113 & 0.0117 \\
\hline & $\gamma_{1}$ & 0.1265 & 0.1252 & 0.0181 & 0.0225 & 0.0227 \\
\hline \multirow[t]{5}{*}{ II } & $k$ & 1.2134 & 1.213 & 0.0182 & 0.0226 & 0.0231 \\
\hline & $\mu$ & 0.0710 & 0.0718 & 0.0048 & 0.0061 & 0.0066 \\
\hline & $\alpha_{1}$ & 0.1833 & 0.1843 & 0.0165 & 0.0202 & 0.0216 \\
\hline & $\beta_{1}$ & 0.2334 & 0.2336 & 0.0117 & 0.0145 & 0.0148 \\
\hline & $\gamma_{1}$ & 0.1732 & 0.1693 & 0.0326 & 0.0418 & 0.0421 \\
\hline \multirow[t]{5}{*}{ III } & $k$ & 1.5139 & 1.5137 & 0.0213 & 0.0274 & 0.0280 \\
\hline & $\mu$ & 0.0740 & 0.0742 & 0.0021 & 0.0026 & 0.0027 \\
\hline & $\alpha_{1}$ & 0.0370 & 0.0352 & 0.0116 & 0.0145 & 0.0152 \\
\hline & $\beta_{1}$ & 0.3436 & 0.3427 & 0.0092 & 0.0119 & 0.0128 \\
\hline & $\gamma_{1}$ & 0 & & & & \\
\hline \multirow[t]{5}{*}{ IV } & $k$ & 1.3632 & 1.3622 & 0.0214 & 0.0268 & 0.0255 \\
\hline & $\mu$ & 0.0584 & 0.0587 & 0.0019 & 0.0024 & 0.0023 \\
\hline & $\alpha_{1}$ & 0.1927 & 0.1922 & 0.0154 & 0.0196 & 0.0185 \\
\hline & $\beta_{1}$ & 0.3220 & 0.3211 & 0.0106 & 0.0138 & 0.0137 \\
\hline & $\gamma_{1}$ & 0 & & & & \\
\hline
\end{tabular}

Table 2: Fitted Int-(G)ARCH models for the period 2006-2011. The number in the parenthesis to the right of the estimate is the associated standard error based on the asymptotic normality.

\begin{tabular}{|c|c|c|c|c|c|c|}
\hline & & \multicolumn{5}{|c|}{ Parameter Estimates } \\
\hline & & $k$ & $\mu$ & $\alpha_{1}$ & $\beta_{1}$ & $\gamma_{1}$ \\
\hline \multirow[t]{10}{*}{ Stocks } & AAPL & $2.1285(0.0420)$ & $0.0038(0.0004)$ & $0.0207(0.0189)$ & $0.4284(0.0186)$ & 0 \\
\hline & AXP & $2.4714(0.0328)$ & $0.0010(0.0001)$ & 0 & $0.4552(0.0092)$ & 0 \\
\hline & $\mathrm{BA}$ & $2.2533(0.0440)$ & $0.0026(0.0004)$ & $0.0525(0.0182)$ & $0.4133(0.0201)$ & 0 \\
\hline & $\mathrm{BAC}$ & $2.2149(0.0433)$ & $0.0002(0.0002)$ & $0.0281(0.0194)$ & $0.5504(0.0164)$ & 0 \\
\hline & DD & $2.4333(0.0469)$ & $0.0017(0.0003)$ & $0.0007(0.0179)$ & $0.4318(0.0174)$ & 0 \\
\hline & JPM & $2.3632(0.0460)$ & $0.0004(0.0003)$ & $0.0133(0.0168)$ & $0.2717(0.0236)$ & $0.4334(0.0493)$ \\
\hline & $\mathrm{KO}$ & $2.5231(0.0484)$ & $0.0012(0.0002)$ & $0.0286(0.0173)$ & $0.3848(0.0167)$ & 0 \\
\hline & MSFT & $2.2515(0.0440)$ & $0.0026(0.0003)$ & $0.0628(0.0205)$ & $0.3931(0.0193)$ & 0 \\
\hline & $\mathrm{T}$ & $2.4932(0.0479)$ & $0.0019(0.0002)$ & 0 & $0.3855(0.0163)$ & 0 \\
\hline & WMT & $2.5040(0.0481)$ & $0.0015(0.0002)$ & 0 & $0.3929(0.0175)$ & 0 \\
\hline \multirow[t]{4}{*}{ Indices } & DJI & $2.4905(0.0485)$ & $0.0007(0.0001)$ & 0 & $0.4331(0.0162)$ & 0 \\
\hline & SPX & $2.3695(0.0465)$ & $0.0007(0.0001)$ & 0 & $0.4418(0.0270)$ & $0.0458(0.0485)$ \\
\hline & FTSE & $2.5773(0.0493)$ & $0.0007(0.0002)$ & 0 & $0.4246(0.0157)$ & 0 \\
\hline & $\mathrm{CAC}$ & $2.1655(0.0422)$ & $0.0012(0.0002)$ & 0 & $0.5036(0.0176)$ & 0 \\
\hline
\end{tabular}


Table 3: In-sample comparisons of Int-GARCH$(1,1,1)$ and $\operatorname{GARCH}(1,1)$. Larger values of $R^{2}$ and smaller values of QLIKE and HMSE indicate higher ranking of the volatility model.

\begin{tabular}{llllllll}
\hline & & \multicolumn{2}{c}{$\mathbf{R}^{2}$} & \multicolumn{2}{c}{ QLIKE } & \multicolumn{2}{c}{ HMSE } \\
Stocks & & GARCH & Int-GARCH & GARCH & Int-GARCH & GARCH & Int-GARCH \\
& AAPL & $\mathbf{0 . 4 7 0 7}$ & 0.3824 & -7.0084 & $\mathbf{- 7 . 0 9 6 8}$ & 0.8020 & $\mathbf{0 . 5 9 7 2}$ \\
& AXP & 0.5099 & $\mathbf{0 . 5 6 9 2}$ & -7.0235 & $\mathbf{- 7 . 1 0 9 8}$ & $\mathbf{0 . 3 6 0 0}$ & 0.4384 \\
& BA & 0.4949 & $\mathbf{0 . 5 4 4 7}$ & -7.2523 & $\mathbf{- 7 . 2 9 9 6}$ & 0.5623 & $\mathbf{0 . 5 1 1 0}$ \\
BAC & $\mathbf{0 . 5 1 7 9}$ & 0.4889 & -6.6973 & $\mathbf{- 6 . 7 9 9 1}$ & 1.1040 & $\mathbf{0 . 9 2 1 1}$ \\
DD & 0.4637 & $\mathbf{0 . 5 7 7 8}$ & -7.2900 & $\mathbf{- 7 . 3 2 9 1}$ & $\mathbf{0 . 5 3 7 4}$ & 0.5583 \\
JPM & 0.3728 & $\mathbf{0 . 4 5 4 5}$ & -6.8266 & $\mathbf{- 6 . 9 2 6 7}$ & 0.6181 & $\mathbf{0 . 5 8 6 1}$ \\
KO & 0.4512 & $\mathbf{0 . 5 2 8 7}$ & -8.2397 & $\mathbf{- 8 . 2 4 6 5}$ & 0.5816 & $\mathbf{0 . 5 3 4 2}$ \\
MSFT & 0.3831 & $\mathbf{0 . 4 3 2 3}$ & -7.4539 & $\mathbf{- 7 . 4 9 3 1}$ & $\mathbf{0 . 8 6 6 4}$ & 1.4878 \\
T & 0.4099 & $\mathbf{0 . 4 5 2 3}$ & -7.5155 & $\mathbf{- 7 . 5 3 9 8}$ & 1.5514 & $\mathbf{1 . 0 6 5 5}$ \\
& WMT & 0.3644 & $\mathbf{0 . 4 0 1 1}$ & -7.8462 & $\mathbf{- 7 . 8 6 9 4}$ & $\mathbf{0 . 7 6 9 4}$ & 1.0324 \\
& & & & & & \\
Indices & DJI & 0.5006 & $\mathbf{0 . 6 6 4 7}$ & -8.5019 & $\mathbf{- 8 . 7 0 5 3}$ & 0.5695 & $\mathbf{0 . 1 8 4 5}$ \\
SPX & 0.5209 & $\mathbf{0 . 6 9 4 2}$ & -8.4010 & $\mathbf{- 8 . 6 2 0 7}$ & 0.4810 & $\mathbf{0 . 2 0 7 4}$ \\
FTSE & 0.4769 & $\mathbf{0 . 6 9 1 1}$ & -8.4324 & $\mathbf{- 8 . 6 4 9 2}$ & 0.4122 & $\mathbf{0 . 2 4 7 8}$ \\
CAC & 0.4171 & $\mathbf{0 . 6 9 1 6}$ & -8.1337 & $\mathbf{- 8 . 3 1 6 2}$ & 0.4158 & $\mathbf{0 . 2 7 5 2}$ \\
\hline
\end{tabular}

Table 4: Comparisons of 1-step-ahead predictions of $\operatorname{Int} \operatorname{GARCH}(1,1,1)$ and $\operatorname{GARCH}(1,1)$.

\begin{tabular}{|c|c|c|c|c|c|c|c|}
\hline \multicolumn{8}{|c|}{ 1-step-ahead Prediction } \\
\hline & & \multicolumn{2}{|r|}{$\mathbf{R}^{2}$} & \multicolumn{2}{|c|}{ QLIKE } & \multicolumn{2}{|c|}{ HMSE } \\
\hline & & GARCH & Int-GARCH & GARCH & Int-GARCH & GARCH & Int-GARCH \\
\hline & & \multicolumn{6}{|c|}{ 1-step-ahead } \\
\hline \multirow[t]{10}{*}{ Stocks } & AAPL & 0.0485 & 0.2929 & -7.5694 & -7.7011 & 0.5426 & 0.4251 \\
\hline & AXP & 0.2591 & 0.5459 & -7.3522 & -7.4381 & 0.3678 & 0.3492 \\
\hline & BA & 0.2909 & 0.4502 & -7.4518 & -7.5154 & 0.3293 & 0.2981 \\
\hline & $\mathrm{BAC}$ & 0.2461 & 0.5466 & -6.6467 & -6.7992 & 0.4693 & 0.4156 \\
\hline & DD & 0.3053 & 0.5000 & -7.3962 & -7.4613 & 0.3275 & 0.3303 \\
\hline & JPM & 0.3587 & 0.3981 & -7.0733 & -7.1928 & 0.3920 & 0.4076 \\
\hline & $\mathrm{KO}$ & 0.2844 & 0.4307 & -8.3893 & -8.4080 & 0.3503 & 0.5011 \\
\hline & MSFT & 0.1032 & 0.1882 & -7.6106 & -7.6670 & 0.7244 & 0.7478 \\
\hline & $\mathrm{T}$ & 0.1530 & 0.2115 & -8.1725 & -8.2027 & 0.4431 & 0.7809 \\
\hline & WMT & 0.2538 & 0.4168 & -6.8641 & -8.4146 & 0.7947 & 0.3420 \\
\hline \multirow[t]{4}{*}{ Indices } & DJI & 0.3204 & 0.7631 & -8.5295 & -8.7353 & 0.3875 & 0.2274 \\
\hline & SPX & 0.3413 & 0.7823 & -8.3776 & -8.5954 & 0.3984 & 0.2411 \\
\hline & FTSE & 0.3421 & 0.7778 & -8.3617 & -8.5712 & 0.4161 & 0.2400 \\
\hline & $\mathrm{CAC}$ & 0.3673 & 0.8003 & -7.8501 & -8.0407 & 0.4095 & 0.2361 \\
\hline
\end{tabular}


Table 5: Comparisons of 2-step-ahead predictions of $\operatorname{Int-GARCH}(1,1,1)$ and $\operatorname{GARCH}(1,1)$.

\begin{tabular}{|c|c|c|c|c|c|c|c|}
\hline \multicolumn{8}{|c|}{ 2-step-ahead Prediction } \\
\hline & & \multicolumn{2}{|r|}{$\mathbf{R}^{2}$} & \multicolumn{2}{|c|}{ QLIKE } & \multicolumn{2}{|c|}{ HMSE } \\
\hline & & GARCH & Int-GARCH & GARCH & Int-GARCH & GARCH & Int-GARCH \\
\hline & & \multicolumn{6}{|c|}{ 2-step-ahead } \\
\hline \multirow[t]{10}{*}{ Stocks } & AAPL & 0.0132 & 0.1517 & -7.5383 & -7.4529 & 0.6343 & 0.4864 \\
\hline & $\mathrm{AXP}$ & 0.1275 & 0.2807 & -7.3106 & -7.3454 & 0.6350 & 0.3145 \\
\hline & $\mathrm{BA}$ & 0.2132 & 0.3081 & -7.4326 & -7.4348 & 0.3797 & 0.3144 \\
\hline & $\mathrm{BAC}$ & 0.1367 & 0.1805 & -6.5818 & -6.585 & 0.8272 & 0.5413 \\
\hline & DD & 0.2097 & 0.3226 & -7.3671 & -7.3819 & 0.4624 & 0.2808 \\
\hline & JPM & 0.2601 & 0.2293 & -7.0329 & -7.0916 & 0.6407 & 0.5443 \\
\hline & $\mathrm{KO}$ & 0.1908 & 0.2436 & -8.3579 & -8.3292 & 0.4782 & 0.4825 \\
\hline & MSFT & 0.0596 & 0.1257 & -7.5891 & -7.5763 & 0.7986 & 0.4576 \\
\hline & $\mathrm{T}$ & 0.0888 & 0.1146 & -8.1397 & -8.1175 & 0.6592 & 0.4360 \\
\hline & WMT & 0.1925 & 0.2579 & -6.7841 & -8.3250 & 0.8143 & 0.3084 \\
\hline \multirow[t]{4}{*}{ Indices } & DJI & 0.2046 & 0.5149 & -8.4939 & -8.5478 & 0.5250 & 0.3641 \\
\hline & SPX & 0.2061 & 0.5574 & -8.3409 & -8.3838 & 0.5378 & 0.3834 \\
\hline & FTSE & 0.2581 & 0.5591 & -8.3375 & -8.3548 & 0.4742 & 0.3965 \\
\hline & $\mathrm{CAC}$ & 0.2666 & 0.5376 & -7.8209 & -7.7907 & 0.4894 & 0.4090 \\
\hline
\end{tabular}

Table 6: Comparisons of 5-step-ahead (one week) predictions of Int-GARCH(1,1,1) and $\operatorname{GARCH}(1,1)$.

\begin{tabular}{llllllll}
\hline \multicolumn{7}{c}{ 5-step-ahead Prediction } \\
\hline & & \multicolumn{7}{c}{$\mathbf{R}^{2}$} & \multicolumn{2}{c}{ QLIKE } \\
Stocks & AAPL & 0.0020 & $\mathbf{0 . 0 1 4 2}$ & $\mathbf{- 7 . 4 9 6 8}$ & -6.8332 & $\mathbf{0 . 6 8 6 1}$ & 0.7015 \\
& & GARCH & Int-GARCH & GARCH & Int-GARCH & GARCH & Int-GARCH \\
\hline & AXP & 0.0278 & $\mathbf{0 . 0 4 4 3}$ & $\mathbf{- 7 . 2 1 4 2}$ & -6.7312 & 1.6016 & $\mathbf{0 . 6 1 4 3}$ \\
BA & 0.1281 & $\mathbf{0 . 1 9 2 3}$ & $\mathbf{- 7 . 3 8 8 7}$ & -7.1597 & 0.5966 & $\mathbf{0 . 4 6 0 7}$ \\
BAC & $\mathbf{0 . 0 6 2 5}$ & 0.0152 & $\mathbf{- 6 . 4 0 1 0}$ & -5.6188 & 3.9111 & $\mathbf{0 . 7 5 8 2}$ \\
DD & 0.1072 & $\mathbf{0 . 1 1 1 0}$ & $\mathbf{- 7 . 2 9 3 8}$ & -6.9044 & 0.9460 & $\mathbf{0 . 5 2 9 7}$ \\
JPM & $\mathbf{0 . 1 6 7 9}$ & 0.1059 & $\mathbf{- 6 . 9 5 1 5}$ & -6.6792 & 1.2665 & $\mathbf{0 . 5 9 1 0}$ \\
KO & $\mathbf{0 . 0 8 7 5}$ & 0.0761 & $\mathbf{- 8 . 2 9 7 7}$ & -7.9787 & 1.2164 & $\mathbf{0 . 5 8 1 2}$ \\
MSFT & 0.0336 & $\mathbf{0 . 0 4 4 3}$ & $\mathbf{- 7 . 5 5 1 0}$ & -7.1817 & 1.0182 & $\mathbf{0 . 5 8 6 1}$ \\
T & 0.0520 & $\mathbf{0 . 0 7 3 1}$ & $\mathbf{- 8 . 0 9 2 9}$ & -7.6682 & 0.9500 & $\mathbf{0 . 5 6 3 8}$ \\
WMT & 0.1068 & $\mathbf{0 . 1 3 0 7}$ & $\mathbf{- 6 . 5 6 0 3}$ & $\mathbf{- 7 . 8 7 4 0}$ & 0.8564 & $\mathbf{0 . 5 5 0 2}$ \\
& & & & & & \\
Indices & DJI & 0.1092 & $\mathbf{0 . 1 2 4 3}$ & $\mathbf{- 8 . 4 0 8 1}$ & -7.9200 & 1.1346 & $\mathbf{0 . 6 4 6 6}$ \\
SPX & 0.1083 & $\mathbf{0 . 1 3 1 5}$ & $\mathbf{- 8 . 2 5 7 1}$ & -7.6855 & 1.1027 & $\mathbf{0 . 6 8 0 1}$ \\
FTSE & 0.1334 & $\mathbf{0 . 1 4 3 9}$ & $\mathbf{- 8 . 2 4 5 5}$ & -7.6797 & 1.1540 & $\mathbf{0 . 6 7 9 6}$ \\
CAC & 0.1696 & $\mathbf{0 . 1 7 9 6}$ & $\mathbf{- 7 . 7 5 5 3}$ & -7.0460 & 0.7602 & $\mathbf{0 . 7 0 7 6}$ \\
\hline
\end{tabular}



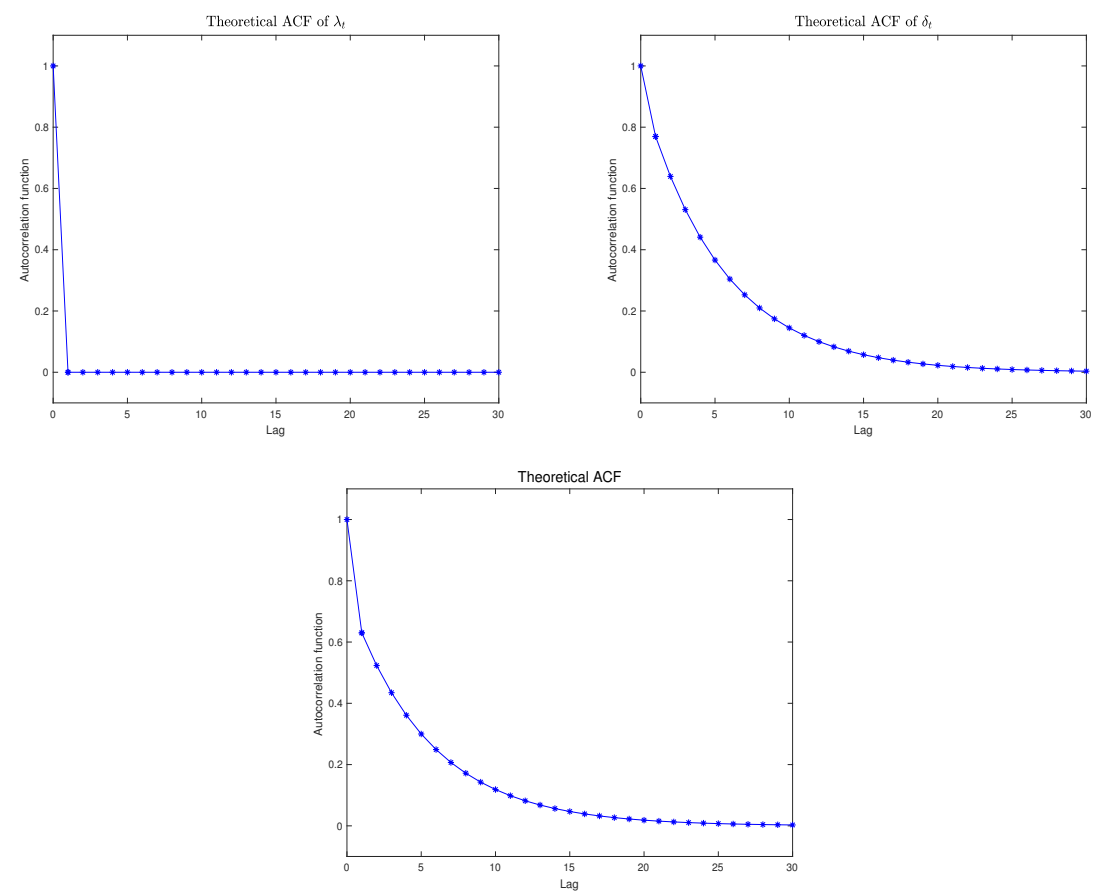

Figure 1: Theoretical auto-correlation functions of Model I. 

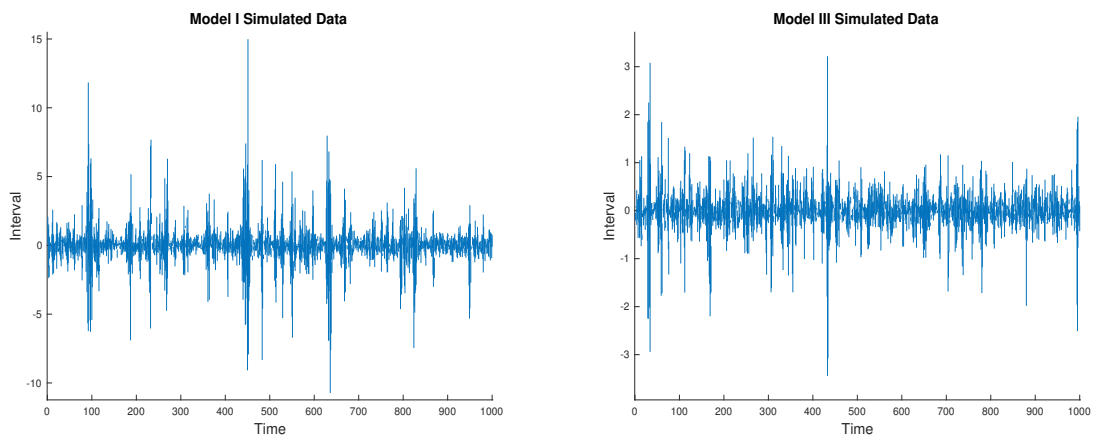

Figure 2: Plots of simulated data sets each with $T=1000$. 

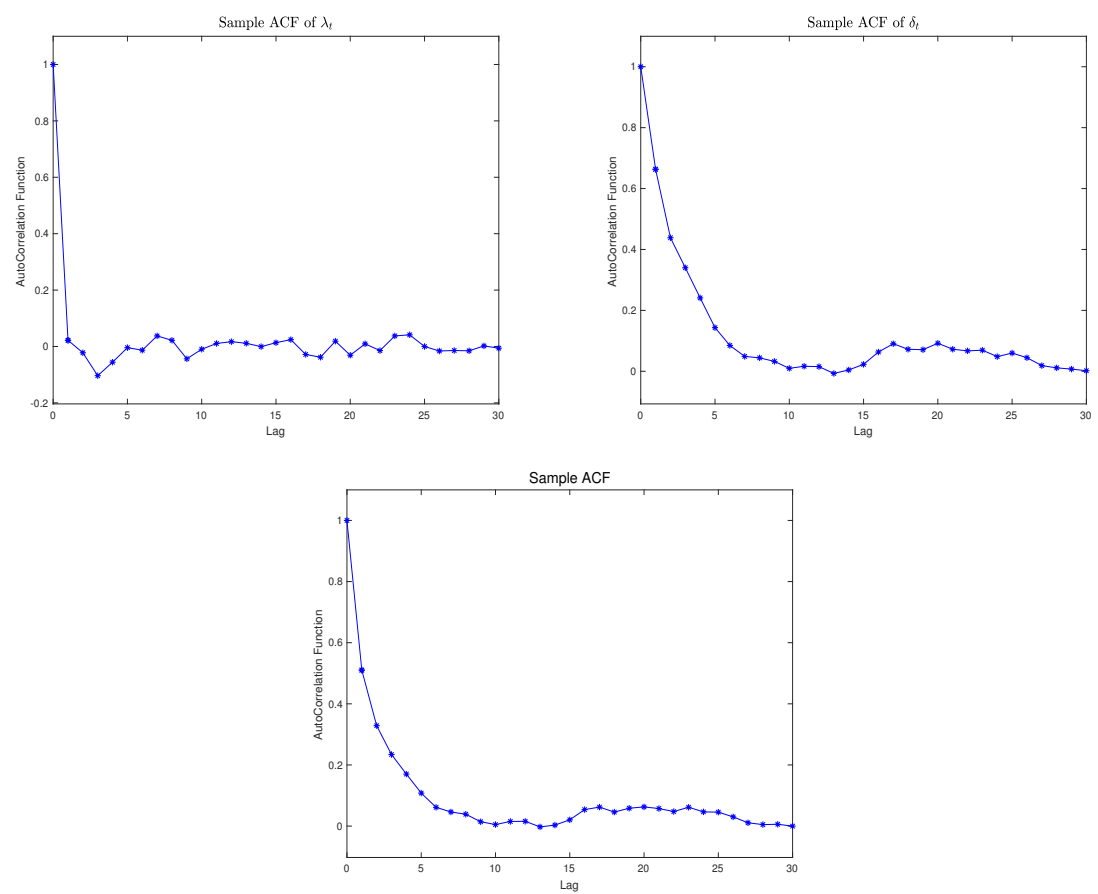

Figure 3: Sample auto-correlation functions of a simulated data set from Model I. 

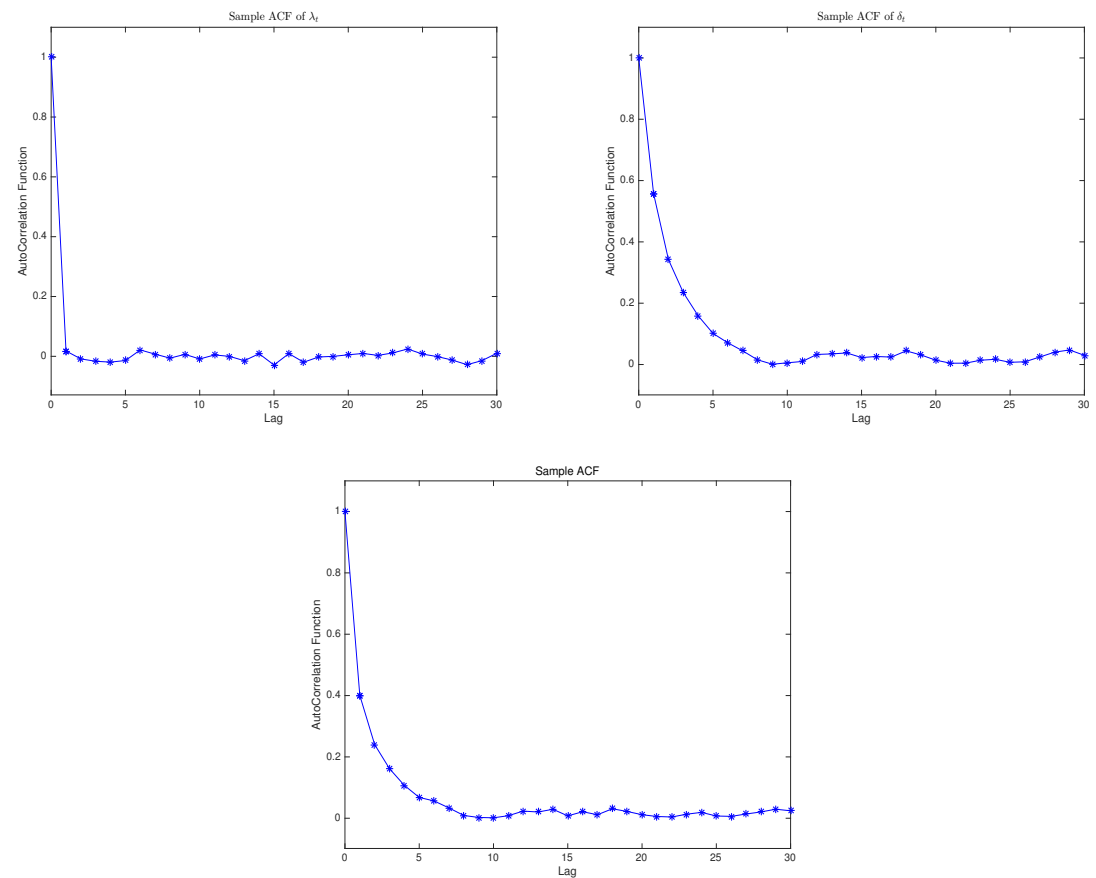

Figure 4: Sample auto-correlation functions of a simulated data set from Model III. 

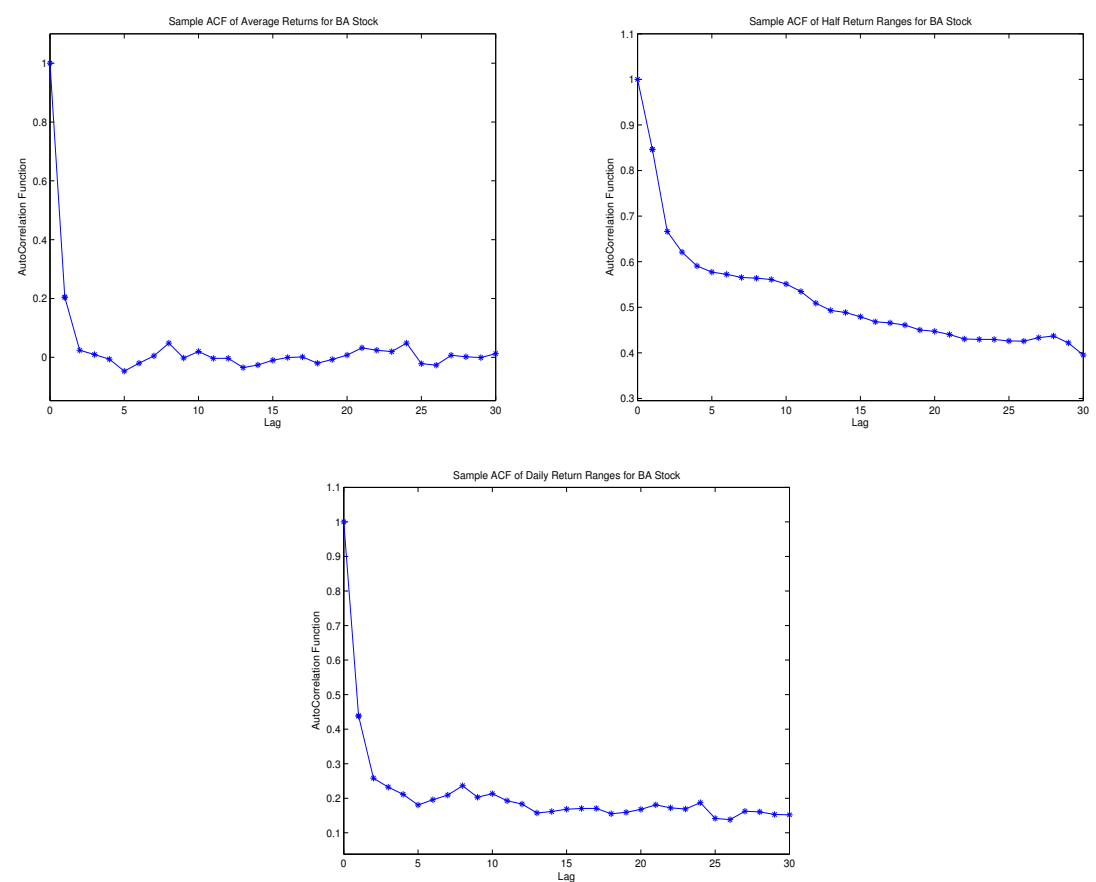

Figure 5: Sample auto-correlation function of BA stock. 